NIST

PUBLICATIONS

\title{
A PROGRAM FOR CALCULATING THE MAXIMUM RADIATION ON A WALL
}

\section{Richard L. Smith}

U.S. DEPARTMENT OF COMMERCE National Institute of Standards and Technology Natlonal Englneerlng Laboratory Center for Fire Research Galthersburg, MD 20899

Supported In part by:

U.S. Alr Force Englneerlng and Services Center, Alrbase Flre Protection and Crash Rescue System Branch Tyndall, AFB, FL 32403-6001 
NATIONAL INSTITUTE OF STANDARDS \& TECHNOLOGY

Research Information Center

Gaichersburg, MD 2039 


\section{A PROGRAM FOR CALCULATING THE MAXIMUM RADIATION ON A WALL}

\section{Richard L. Smith}

U.S. DEPARTMENT OF COMMERCE Natlonal Instltute of Standards and Tochnology National Englneoring Laboratory Center for Fre Research Galthersburg, MD 20899

Supported In part by: U.S. Alr Force Englneering and Services Center, Alrbase Fire Protection and Crash Rescue System Branch Tyndall, AFB, FL 32403-6001

October 1990

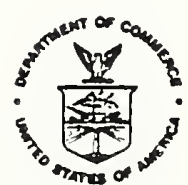



TABLE OF CONTENTS

Abstract

Forward

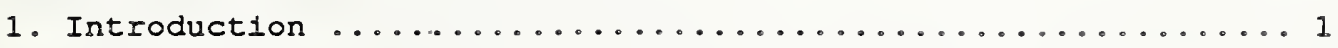

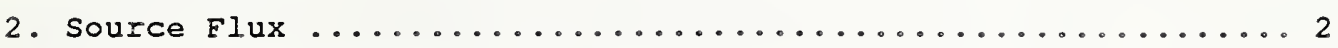

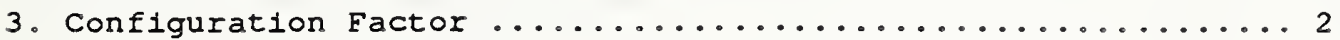

3.1 Program Input and Basic Functions ............ 4

3.2 Configuration Factor of an Element ........... 7

3.3 Shortest Distance Point to Planar Region ....... 7

3.4 Number of subelements in opening ............. 9

3.5 Configuration Factor for one Opening .......... 10

3.6 Configuration Factor for $\mathrm{N}$ Openings ........... 11

4. Determining Locations and Strength of Maximum

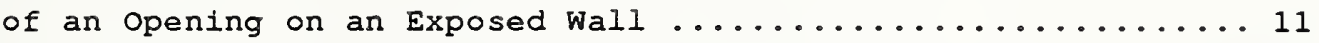

5. Determine Area to Scan for Maximum ................... 13

6. Maximum Configuration Factor for each subregion of the

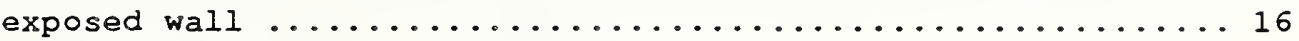

7. Conclusions and Possible Future work .....................

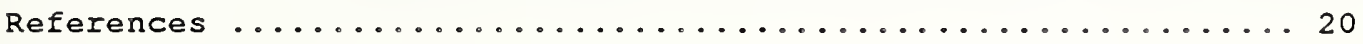

Appendices

Appendix A: Configuration Factor Calculation Compared to

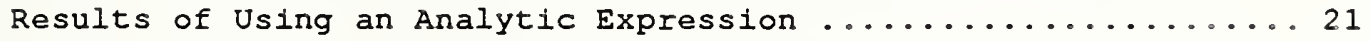

Appendix B: Results for some simple Buildings .............25 


\begin{abstract}
This report describes a program module of the expert system EXPOSURE. This module, written in Common Lisp, is for calculating the maximum electromagnetic radiation incident upon a building's wall due to the burning of a neighboring building. It is assumed that the burning building has an arbitrary number of rectangular openings emitting radiation. The exposed wall can be considered as being composed of a number of rectangular regions. These regions may be openings or regions of different materials. This program can determine the maximum radiation for each of these regions and for the wall less these regions.
\end{abstract}

Keywords: exposure, radiation, radiative heat transfer, computer programs. 
In this report we are trying a somewhat unusual approach to documenting a computer program. This entire report is the contents of the computer file that contains the program "Radiation." The only things omitted are some semicolons that indicate that the written texts of this report are comments and the initial header strip:

;; -*- Mode: Joshua; Package: FIRE-PROTECTION; Syntax: Joshua; Default-character-style: $\because ;$ (:FIX :ROMAN :LARGE) -*- Created 7/27/89 16:15:45 by Smith running on SMOKEY at $\because$ NBS.

The only addition to this report that is not in the file is the figure. A computer program is a dynamic thing and should be documentated during development as well as when it is finished. This report was generated by printing a file which is a module in an expert system. It represents a snap shot of the module at one instance of time. Since the computer code is the focus of this report it is included in the body of the report. The document is designed to be read in its entirety although the audience wanting to do so is probably small. We believe this type of documentation is required for modules of expert systems which are going to be used to make real world recommendations. This type of document will allow others to review, revise, and maintain this code and its physical science basis. Those who do not understand LISP code can skip reading the code. which is printed in bold type style, enclosed in parentheses, and often starts with def... . These readers should be able to follow the logic of the program but they may not be able to determine if the code performs as described. 



\section{Introduction}

This report documents a module of the expert system EXPOSURE. It describes a program for calculating the maximum electromagnetic radiation incident upon a building's wall due to the burning of a neighboring building. It is assumed that the burning (exposing) building has an arbitrary number of rectangular openings emitting radiation. The exposed wall can be considered as being composed of a number of rectangular regions. These regions may be openings or regions of different materials. We wish to determine the maximum radiation for each of these regions and for the wall less these regions.

After rescuing people from a burning building, the next highest priority item for fire fighters is to prevent the fire from spreading to adjacent buildings [1]. Because of the potential damage that can be caused if fire propagates from building to building there are standards that provide guidance on how to reduce the likelihood of fire spreading [2] [3].

A critical part of the analysis of the spread of fire between buildings is the calculation of the electromagnetic radiation falling upon the exposed surfaces of the nonburning building. The calculations of the radiation is complex and does not lend itself to simple approximations [5] [6] [7] [8]. Present standards are based on work done before the advent of the personal computer [4] [5]. To make the estimating of the radiation level in the standards possible, a number of simplifying assumptions had to be made in the existing standards.

NFPA 80A, Recommended Practice for Protection of Buildings from Exterior Fire Exposures [2], makes a number of assumptions. Some are used in this expert system others are not. We do make the following assumptions which are also made by NFPA 80A:

1. Only one wall of the burning building will expose one wall of the exposed building.

2. All radiating openings are assumed to have a rectangular shape [5].

We do not make the following assumptions that are made by the NFPA 80A.

1. The exposed and exposing walls are parallel (for an exception see [3]).

2. A region of a wall with a number of radiators can be treated as a single radiator with a size of the rectangle which encloses all the individual openings and which radiates at a reduced intensity. The reduced intensity equals the ratio of the area of the openings to that of the enclosing rectangle.

3. If the exposed building has a combustible wall, then the minimum allowed separation between buildings is the separation such that the thermal radiation falling on the exposed building just equals $1.26 \mathrm{w} . / \mathrm{sq}$. $\mathrm{cm}$. (the piloted ignition level for oven dried wood).

4. Only the maximum radiation level on the exposed wall is determined.

Briefly, this program does the following: Based upon the size of an opening and its minimum distance from the exposed wall, the program divides the opening into subelements. It then finds the configuration factor due to one opening by summing the contributions from all the small elements of the area in the opening. It then sums all the contributions from all the openings. This gives the configuration factor at any point on the exposed wall due to all the openings. The program then can determine the maximum configuration factor in any rectangular region on the exposed wall by scanning the region. Finally, it has the capability of finding the maximum configuration on the area of the exposed wall less its openings.

The program described in this report was written in Symbolics Common LISP. (Note: Certain commercial products are identified in this report in order to adequately specify the computer program. Such identification does not imply recommendation by the National Institute of Standards and Technology, nor does it imply that these products identified are necessarily the best available for the purpose.) 


\section{Source Flux}

We assume all surfaces of openings in the exposing building radiate according to the equation

$$
E=\varepsilon \lambda T \wedge \mathrm{w} / \mathrm{sq} \mathrm{cm} .
$$

where $\varepsilon$ is the emissivity ( $\varepsilon \leq 1$ ). For an ideal black body $\varepsilon=1$ and where $\lambda$ is the Stefan Boltzmann constant with a value of $5.6703 \mathrm{e}-12$ (w./sq. $\mathrm{cm}$.) $(\mathrm{K})^{\wedge}-4$. We will assume $\varepsilon=1$ for all the openings. Since a real opening will always radiate less than a black body at the same temperature, this assumption results in overestimating the radiation and therefore errs on the side of safety.

We record the Stefan Boltzmann constant in the program as follows:

(defconstant *Stefan-Boltmann-constant* $5.6703 a-12$ "(w/sq cm) (K)^-4")

The LISP function black-body-flux is the LISP version of eq. 1. It takes as its input or its parameter the temperature of the burning compartment. It returns the radiation flux at the surface of the openings of the building in $\mathrm{w} . / \mathrm{sq} . \mathrm{cm}$.

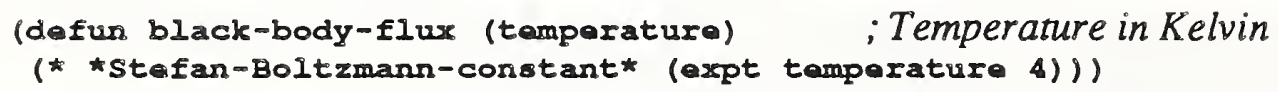

\section{Configuration Factor}

If we assume Lambert's cosine law holds, the incident radiant flux falling on a small element of surface is

$$
R f=E \int(\cos \alpha 1 \cos \alpha 2) /\left(\pi r^{\wedge} 2\right) d A 1
$$

where we integrate over the surface $A 1$, and $E$ is the source flux determined above. The integral is called the configuration factor. From Figure 1. we see that $r$ is the distance between the point of integration at $d A 1$ to the point at which we are evaluating the field, $\alpha 1$ is the angle between the normal to $\alpha_{A} I$ (the exposing surface) and $r$, and $\alpha 2$ is the angle between the normal to the exposed surface at the point we are evaluating the field and $r$. If $(x a, y a, z a)$ is the source point on $d A I$ and $(x 0, y 0, z 0)$ is the field point on the exposed surface, then we have

$$
I=\left((x 0-x a)^{\wedge} 2+(y 0-y a)^{\wedge} 2+(z 0-z a)^{\wedge} 2\right)^{\wedge} .5
$$

If we define $\gamma$ and $\beta 1$ such that

$$
\begin{aligned}
& \cos \gamma=\left(x_{0}-x a\right) / r \\
& \sin \gamma=\left(y^{0}-y a\right) / r \\
& \cos \beta 1=\left(x^{2}-x 1\right) / L 1 \\
& \sin \beta 1=\left(y^{2}-y 1\right) / L 1
\end{aligned}
$$

where $\beta_{1}$ is the slope of wall (the exposing wall) and the length of the wall is given by

$$
L 1=\left(\left(x^{2}-x 1\right)^{\wedge} 2+\left(y^{2}-y 1\right)^{\wedge} 2\right)^{\wedge} .5
$$

then we have 


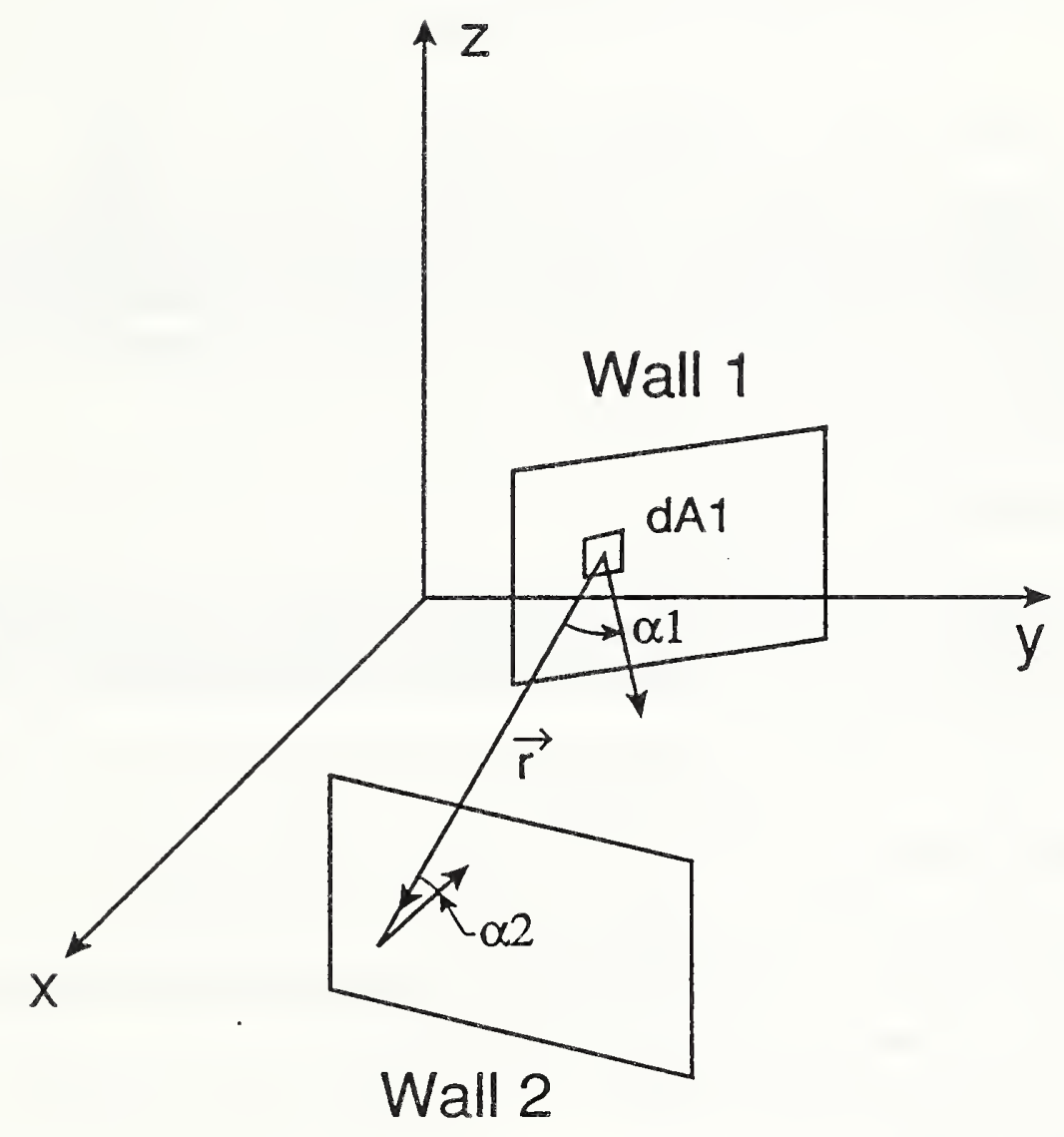

Figure 1.0 Variables of the Configuration Integral 
If we define $\beta 2$ such that

$$
\begin{aligned}
& \cos \beta 2=\left(x^{4}-x^{3}\right) / L 2 \\
& \sin \beta 2=\left(y^{4}-y^{3}\right) / L 2
\end{aligned}
$$

where $\beta 2$ is the slope of wall 2 and the length of exposed wall (wall2) is given by

$$
L 2=\left(\left(x^{4}-x^{3}\right)^{\wedge} 2+\left(y^{4}-y^{3}\right)^{\wedge} 2\right)^{\wedge} .5
$$

then we have

$$
\cos \alpha 2=\cos \gamma \sin \beta 2-\operatorname{sim} \gamma \cos \beta 2 \text {. }
$$

All rectangles are defined by giving the coordinates of the lower left hand corner and the upper right hand comer. This is sufficient to define a rectangle because all rectangles of interest are either vertical or horizontal. Therefore, an opening or a wall can be defined by the four points: $(x 1, y 1, z 1),(x 2, y 2, z 1),(x 2, y 2, z 2)$, and $(x 1, y 1, z 2)$ or by using the two point convention, $(x 1, y 1, z 1)$

and $(x 2, y 2, z 2)$.

\subsection{Program Input and Basic Functions}

The input for this program is the lower left and upper right corners of the exposing wall and its openings and the exposed wall and its openings. Important concepts for this program are various types of points and rectangles. In this section we give their definitions and the basic functions for operating on them.

One basic entity is the point which we define as:

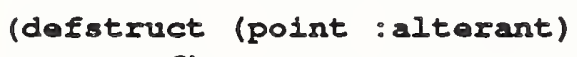

Sometimes we have an interest in the configuration factor for a point so it has been included. Another basic entity is the vertical rectangle which we define as:

(defatruct (vertical-rectangla)

lowar-left upper-right)

Then walls, openings, and elements of integration are just instances of vertical rectangles.

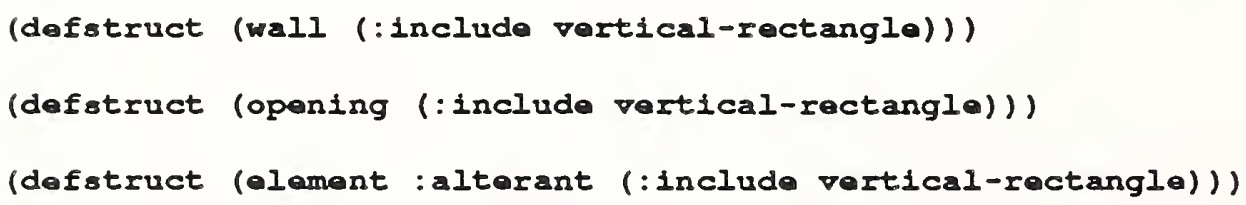

We will have use for two simple functions. One is the square-root and the other is the square. We define these functions as follows:

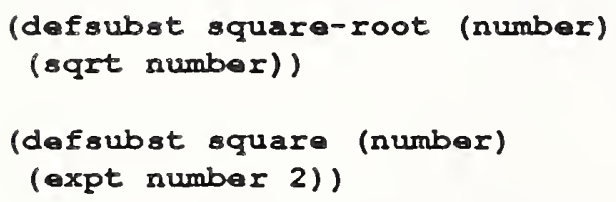

We used defsubst so they get expanded at compile time. 
We will have use for a function which rounds off some of our results to a controllable number of significant figures. We call that function round-off.

(dafun round-off (number coptional (significant-figuras 0))

(lot ((d (axpt 10 significant-figures)))

(/ (round (* number d)) d)) )

We will have need to determine the separation between two points.

(defun point-separation (point-1 point-2) ; dimensions

(square-root

(t (square (- (point-x point-2) (point-x point-1)))

(square (- (point-y point-2) (polnt-y polnt-1)))

(square $(-($ point-z point-2) (point-z point-1))))))

We use point-separation to determine the length of the diagonal of vertical rectangles.

(defun vertical-ractangle-diagonal (vertical-rectangla)

(point-separation (vortical-rectangle-lowar-left vertical-rectangle)

(vertical-rectangle-upper-right vertical-ractangle)))

We will also need the separation of the projection of two points on the xy-plane.

(dofun $x y-s e p a r a t i o n ~(p o i n t-1$ point-2) ;separation in $x y$ plane

(square-root

(t (squara (- (point-x point-2) (point-x point-1)))

(square $(-($ point-y point-2) (point-y point-1))))))

We use $x y$-separation to determine the width of vertical rectangle.

(defun vertical-ractangle-width (vartical-ractangle)

(xy-separation (vertical-rectangle-lower-left vertical-rectangle)

(vertical-rectangle-upper-right vertical-rectangle)))

Instead of calling the wall dimension in the xy plane its width, we called it length.

(defun wall-langth (wall)

(vartical-ractangle-width wall))

;note switch form length to width

But for an opening we switch back to width.

(defun opening-width (opening)

(vertical-ractangle-width opening))

We will need the height of our rectangles so we define the following:

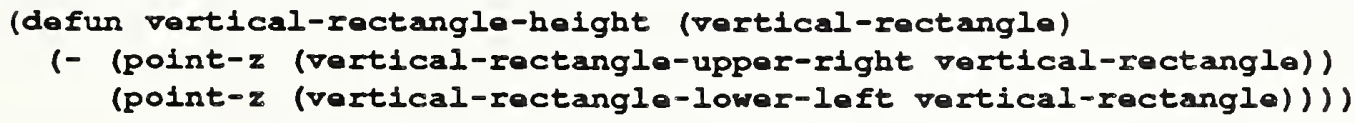

Likewise, we will need the area of openings and elements of integration.

(defun vertical-rectanglo-area (vertical-rectanglo)

(* (vertical-rectangle-height vertical-rectangle)

(vertical-rectangle-width vertical-rectangle))) 


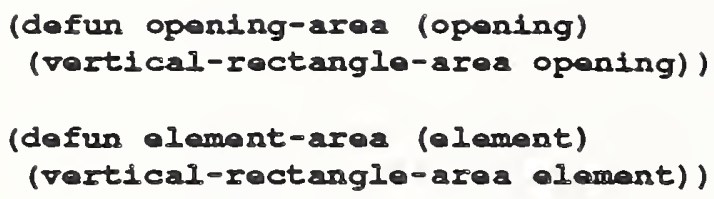

The following functions are used to calculate the coordinates of the centers of various rectangles.

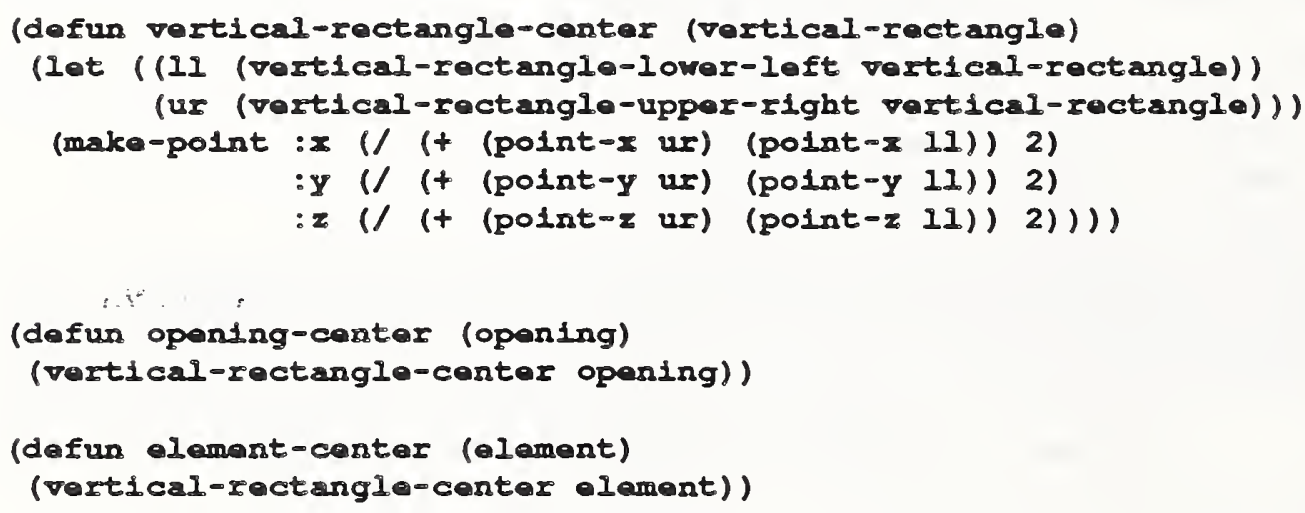

The slope of the various rectangles in the xy-plane can be determined by these functions.

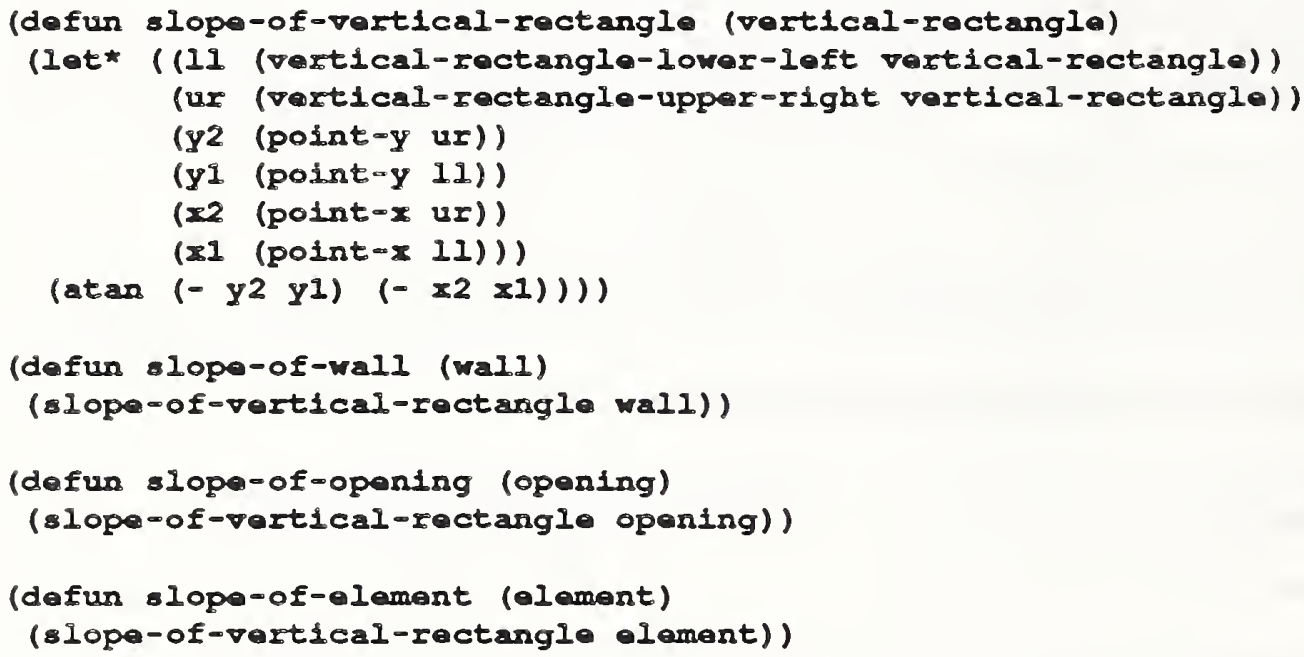

This function determines the cosines of the alpha angles.

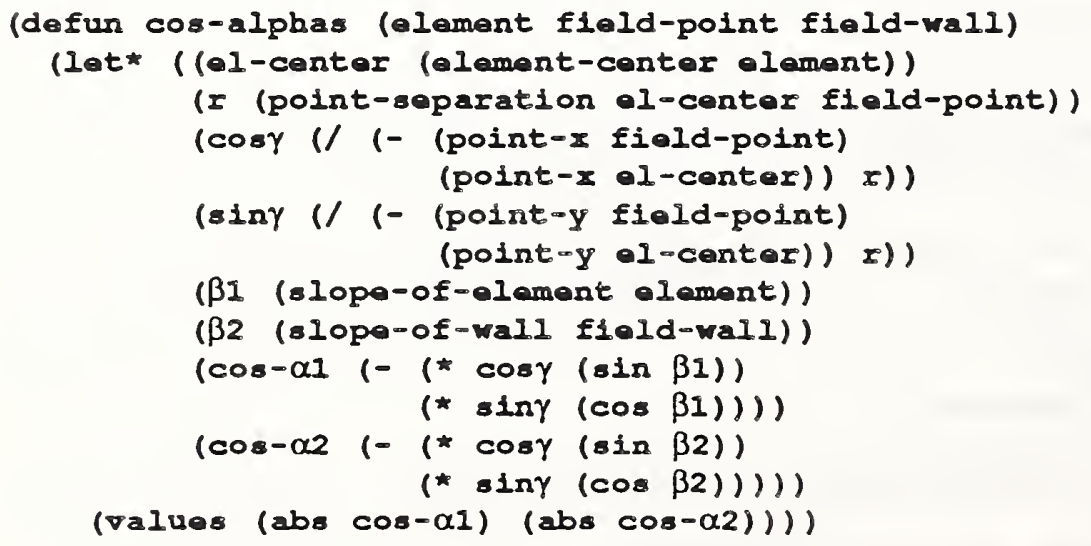




\subsection{Configuration Factor of an Element}

Whenever we have the value of the diagonal, $d$, of an element of an opening much less than the value of the shortest value of $r$ over the opening, i.e., $d \ll r-m \pm n$ where

$$
d=\left(\left(x_{2}-x_{1}\right)^{\wedge} 2+\left(y^{2}-y_{1}\right)^{\wedge} 2+\left(z 2-z_{1}\right)^{\wedge} 2\right) \wedge .5
$$

then $\alpha 1, \alpha 2$, and $I$ do not vary appreciably over the element of area $A 1$.

Therefore, we can approximate the configuration integral for one element by the following expression:

$$
c f=(\cos \alpha 1 \cos \alpha 2) A 1 /\left(p 1 \quad I^{\wedge} 2\right)
$$

We will define $\mathrm{p} 1$ to be 3.141593 . This avoids double precision calculations and thereby speeds up the computations.

$$
\text { (defrar *single-p-p1*3.141593) }
$$

We now define the LISP function configuration-factor-for-one-element with parameters of element, fleld-point, and field-wall. It returns the numerical value for the configuration factor for this infinitesimal element at the field-point.

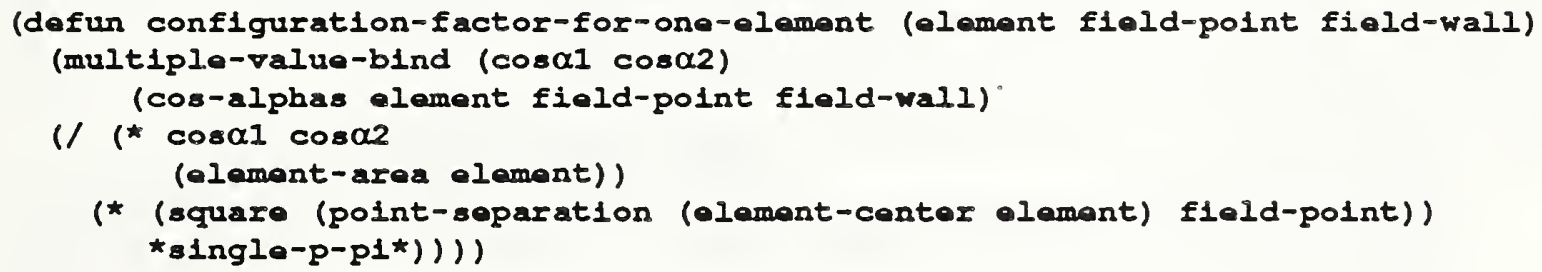

We have found that if we have $d \leq .1 \star r-m 1 n$, we do not need to integrate over the area with this value of $d$. We get a good approximation for our integral by using conflguration-factor-for-one-elemont. Therefore we define our constant *limit-elament-diagonal-to-r-min-ratio* as follows

(defvar *Ilmit-element-diagonal-to-r-min-rat1o* . 1)

If our area of interest does not satisfy this condition, we must subdivide it into subelements until it does.

To implement this approximation we need to be able to 1.) calculate the shortest distance between a point and a planar region $(r-m i n)$ and 2.) determine the number of subelements so we can use the above approximation to calculate the configuration factor.

\subsection{Shortest Distance Point to Planar Region}

We wish to determine the shortest distance between a point $(x 0$ yo $z 0)$ and a planar rectangular region defined by its coordinates of opposite corners $\left(\begin{array}{lll}x 1 & y 1 & z 1\end{array}\right)$ and $\left(\begin{array}{lll}x^{2} & y^{2}\end{array}\right.$ $z 2$ ). We assume the region lies in a plane that is perpendicular to the xy plane, i.e. the vertical plane.

The inputs for the function shortest-distance-point-to-plane-region are the point and vertical-rectangle. It returns the shortest distance and the coordinates of the nearest point in the rectangle. 


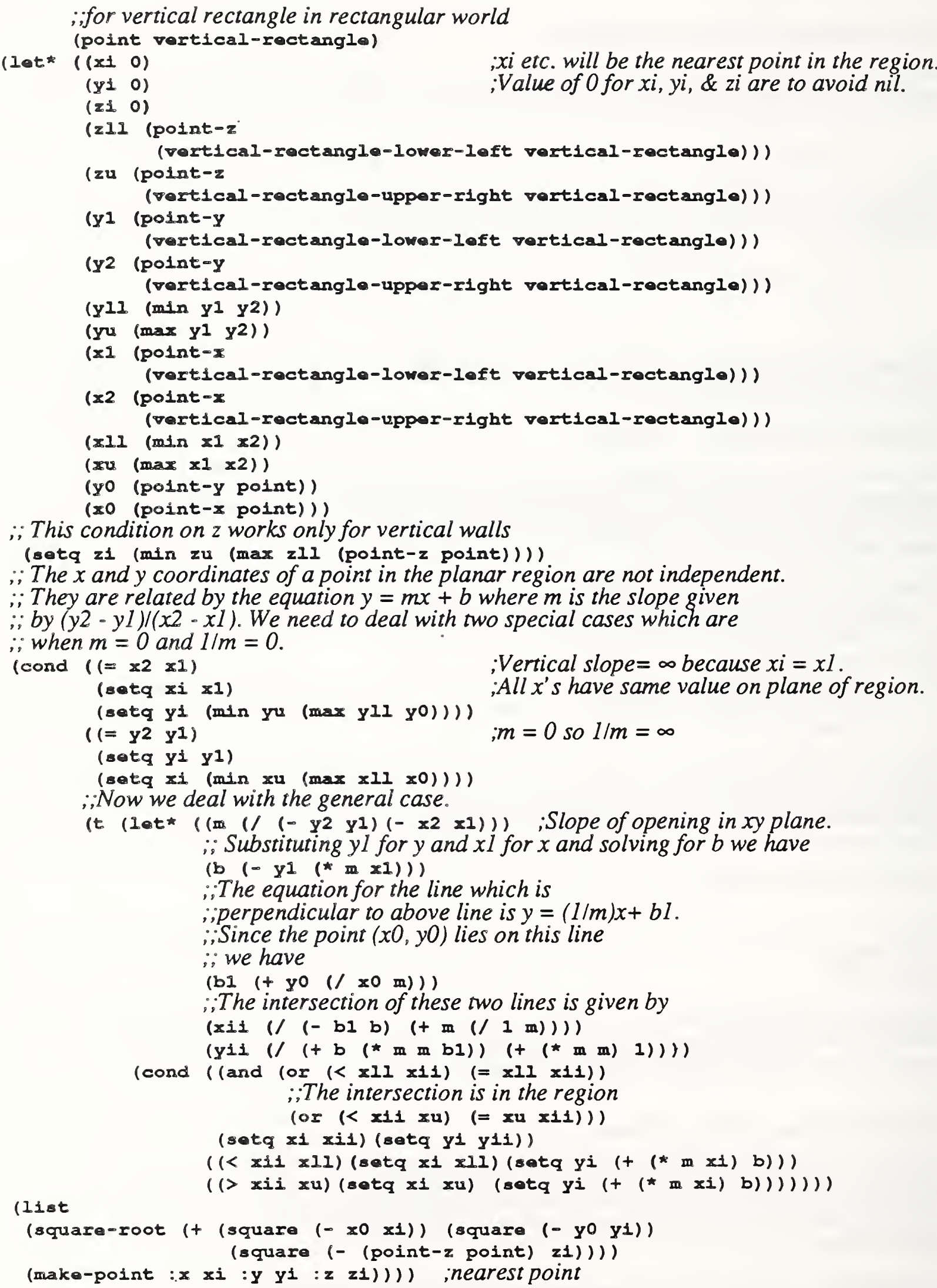




\subsection{Number of Subelements in Opening}

We have an opening with sides longor, $L$, and shorter, $s$, we want the diagonal to be less than *limit-alament-diagonal-to-r-min-ratio* times the minimum value of the distance from the field point and any point in the opening. If

$\left(\left(L^{\wedge} 2+s^{\wedge} 2\right)^{\wedge} .5\right)<\left(* I 1 m 1 t-a l a m a n t-d i a g o n a l-t o-r-m i n-r a t 10 *{ }^{*} r-m i n\right.$,

then we are done. However, if this is not the case, we need to subdivide the opening into approximate square elements. Therefore, we let $n=L / s$, then if we divide $L$ by $n$ we get square elements. If we divide $L$ by $N=$ (round $n$ ) we get nearly square elements. If

$\left((L / n) \wedge 2+s^{\wedge} 2\right)^{\wedge} .5<(*$ limit-element-dlagonal-to-r-min-ratio*)*I-min,

we are done. Otherwise, we must continue making our elements smaller by reducing the size of both dimensions by dividing them by $p$.

Then we want an integer $P$ such that

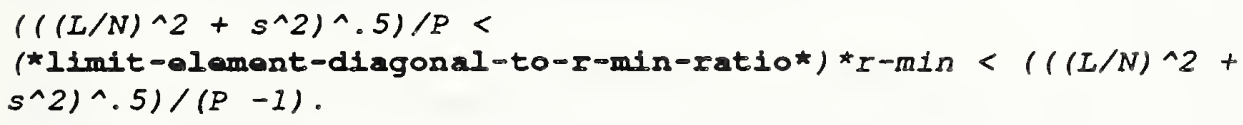

This can be achieved as long as $r-m i n$ is not zero. If $r-m i n$ is small, $P$ may become so large as to make this approach undesirable.

We make

$p=\left(\left((L / N)^{\wedge} 2+s^{\wedge} 2\right)^{\wedge} .5\right) /(\star 1$ Imtt-oloment-diagonal-to-r-min-ratio*)*r-min.

It follows that $P=$ (ceiling $p$ ). We rote that (ceiling $p$ ) is a LISP expression which returns an integer which equals $p$ if $p$ is an integer otherwise it returns the first integer larger than $p$.

We recall that LISP can't tell the different between $N$ and $n$.

The function subelements-paramoters takes the width and height of the opening and $r-m i n$ as input and retums a list which has the form:

(divisor-of-width divisor-of-height).

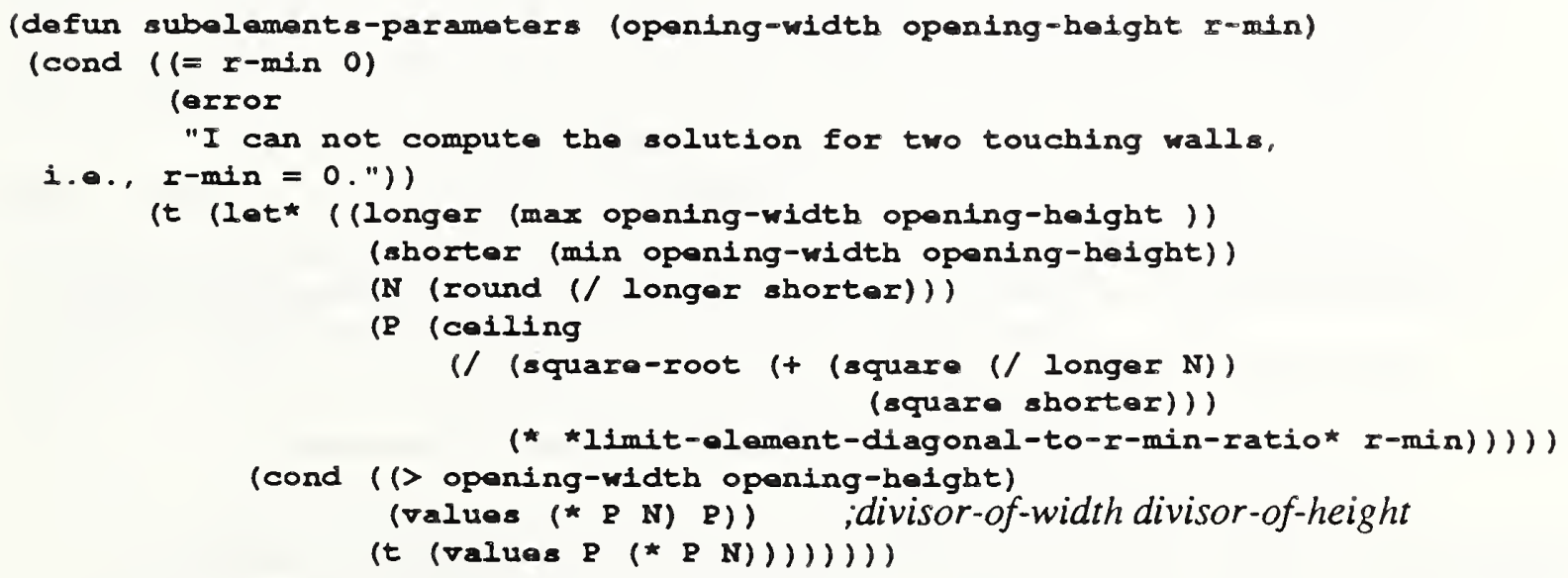




\subsection{Configuration Factor for One Opening}

To obtain the configuration factor for one opening, we sum the configuration factor for all the subelements in that opening. The function configuration-factor-for-one-opening take as input the field-polnt, opening, and exposed-wall. It returns the configuration factor of the opening at the field-point.

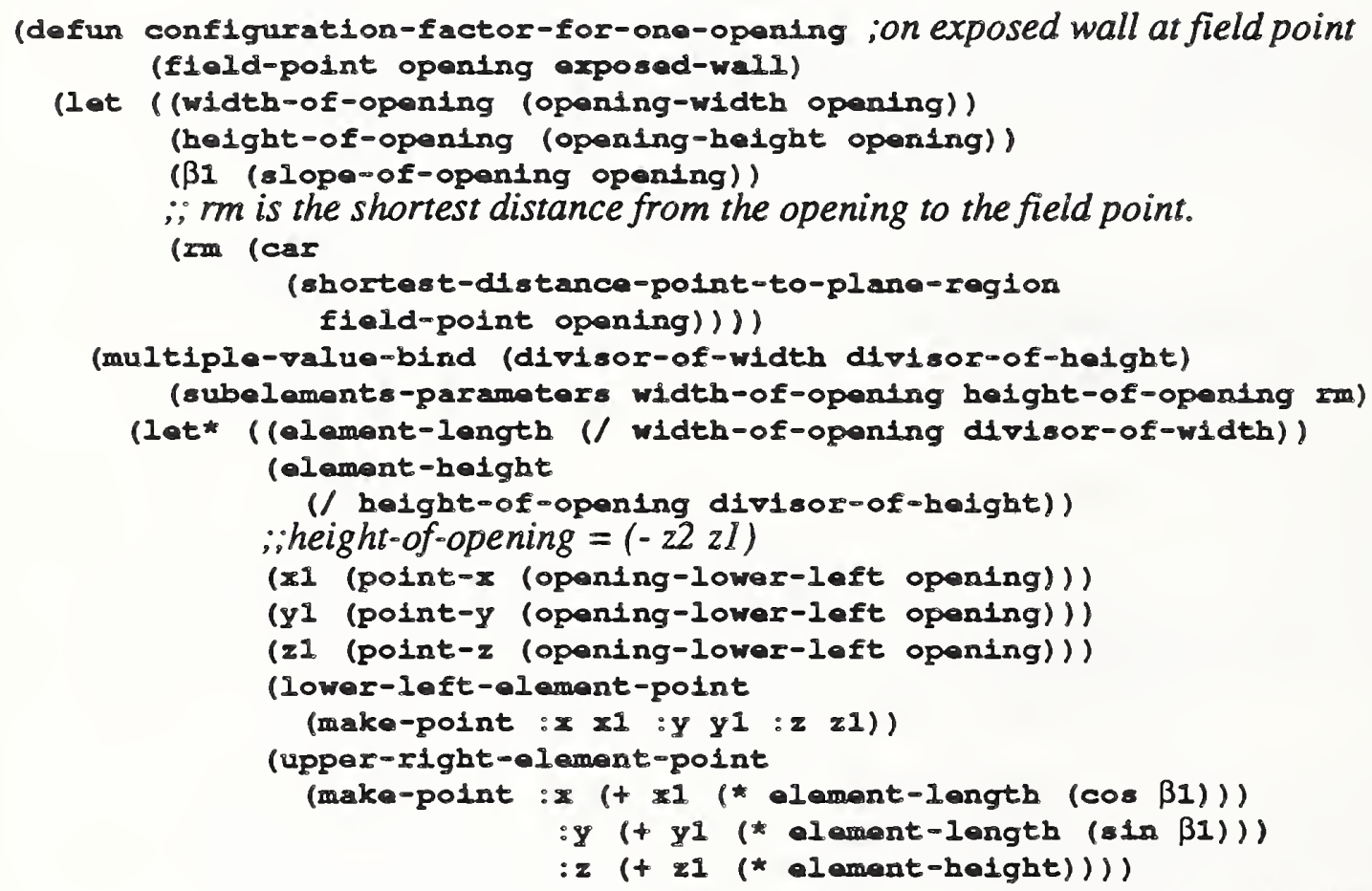

七)))

(loop for 1 from 0 to (- divisor-of-height 1)

;loop over elements in opening

with sum $=0$

do

(altax-point lowex-left-element-point z (t z1 (* 1 element-baight)) )

(alter-point upper-right-alement-point z (t z1 (* (t 1 l) element-height))

(loop for $j$ from 0 to (- divisor-of-width 1 )

do

(alter-point lower-left-alament-point $x(+x 1$ (* j element-length (cos $\beta 1)$ )

)

os $\beta 1)$ ))

$$
\left.y\left(+y^{1}(* j \text { alament-longth }(\sin \beta 1))\right)\right)
$$

(altar-point upper-right-alement-point $\times(+x I(*)(t j 1)$ element-length (c

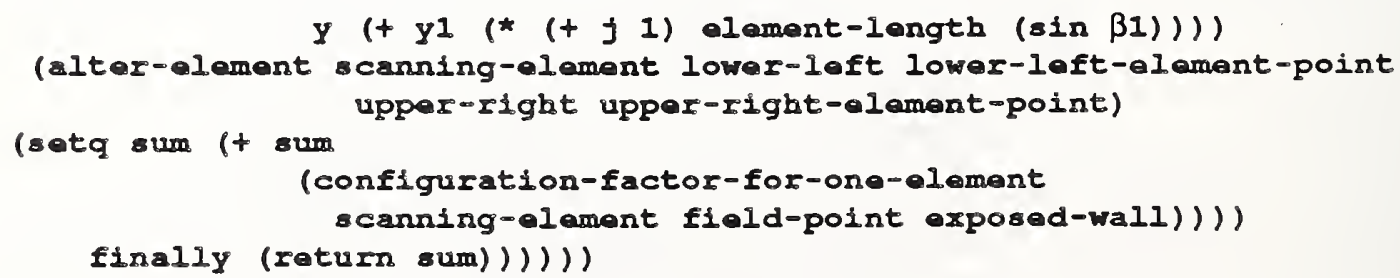

Comparing the results of configuration-factor-for-one-opening with analytical expressions for three special cases showed agreement to 4 or 5 decimal places (see Appendix A). Note that this is an approximate integration which depends upon such things as the value

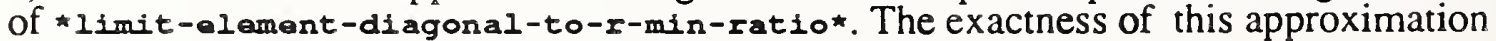
can be improved by the proper adjustment of this parameter. 


\subsection{Configuration Factor for $\mathrm{N}$ Openings}

If we have an arbitrary number of openings, we sum the configuration factors for all the individual openings. The function configuration-factor-for-n-openings has input of the flald-point, list-of-openings, and exposed-wall It returns the configuration factor due to all the openings at the field point.

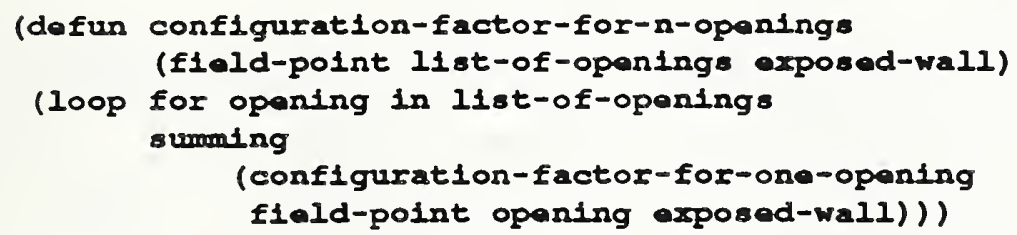

At this point we can calculate the field at any point on the exposed wall.

\section{Determining Location and Strength of Maximum of an Opening on an Exposed Wall}

The next step is to determine the locations and strengths of the maximum configuration factor (or field) on an exposed wall due to the radiation from one opening in the exposing wall.

If we have a group of openings for which the separation between openings is much smaller than the shortest distance to the exposed wall, then such a block of openings can be treated as a single opening whose area is the sum of the individual opening's areas.

It can be shown that the maximum for an element of an opening will lie on the exposed wall at the point closest to the center of the element.

To find the the maximum of an opening we will start by guessing its location. Then we will search for a point that has a configuration factor that is close to the maximum configuration factor. Close means within $2 \%$ or less. So we need to pick a point for our initial guess and the size of steps we will take while searching for our maximum.

Because of symmetry in the $z$ direction, the maximum for an opening will always have a $z$ value equal to the midpoint height of the opening if it falls in the exposed rectangle of interest. In addition, the maximum of an opening frequently lies at the point which is nearest the center of the opening. This tends to be a better guess the smaller the alpha angle. However, it is still reasonable even out to 45 degrees. Our first guess for the location for the maximum is at the point nearest the center of the opening. Then we try to walk to a greater value for the configuration factor. We move in a plane parallel to the $x y$ plane a distance given by the step size and see what the configuration factor is at that point.

The function step-size takes as input the oponing and the axposed-wall. To estimate the step-size we assume that the relative change in the configuration factor goes as (step-size/separation $)^{\wedge} 2$ which is characteristic of an inverse square relationship where the change is approximately perpenticular to $r$. We believe this is a conservative assumption. Significant improvement in the code's speed may be achieved by an improvement here.

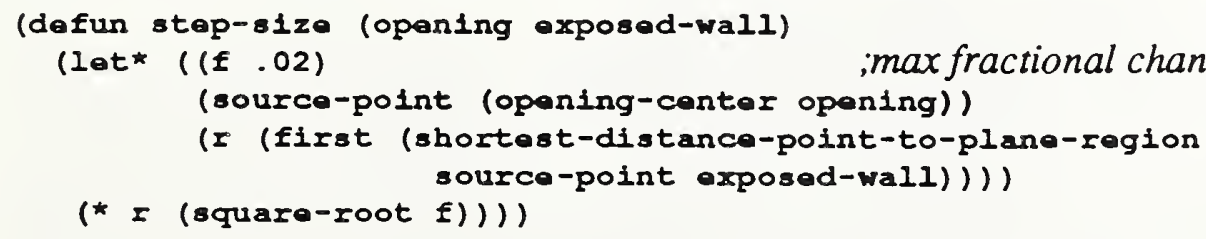

The function Maximum-of-an-Opening takes as input the opening and exposed-wall 
and returns the field-point (the location of the maximum for the opening) with the cf slot having a value of cfmo (the maximum configuration factor for the opening).

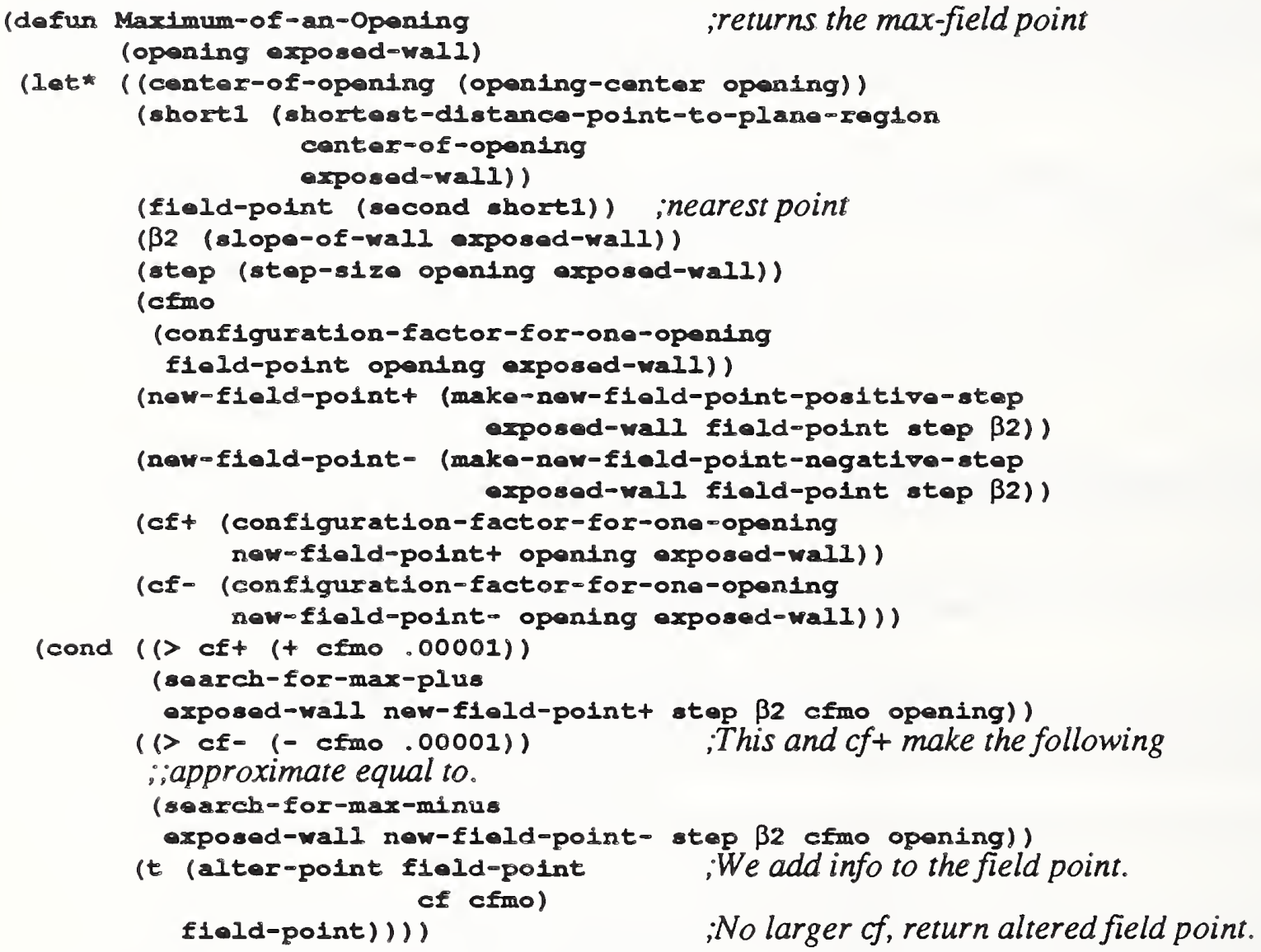

We first try to move in a positive $\mathrm{x}$ or $\mathrm{y}$ direction. If we don't find a maximum, we look in the negative direction. We note that for one opening the configuration factor is monotonic to its maximum. We take a step by making a new field point either in the positive or negative direction. We first write the function for a positive step.

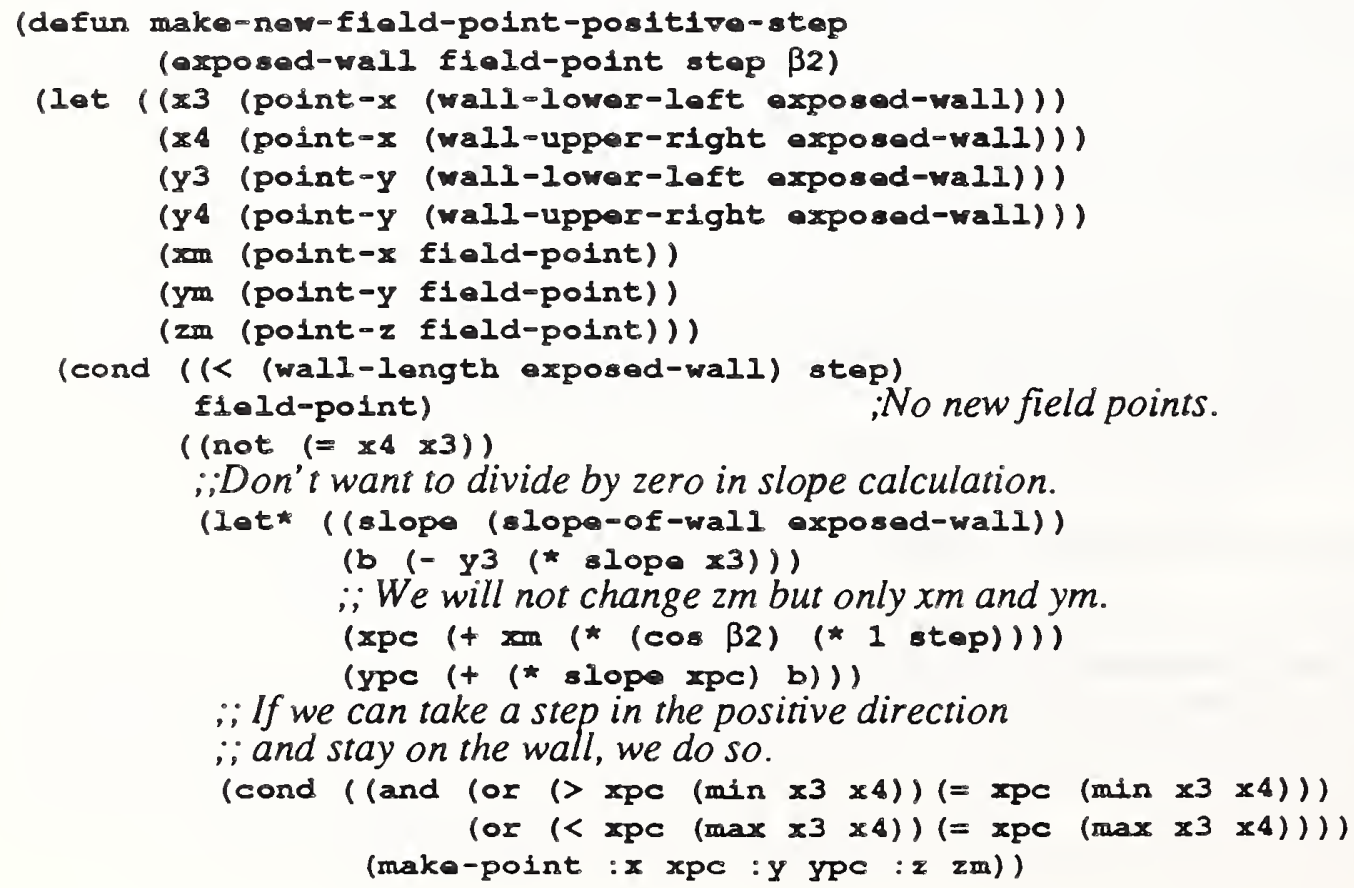




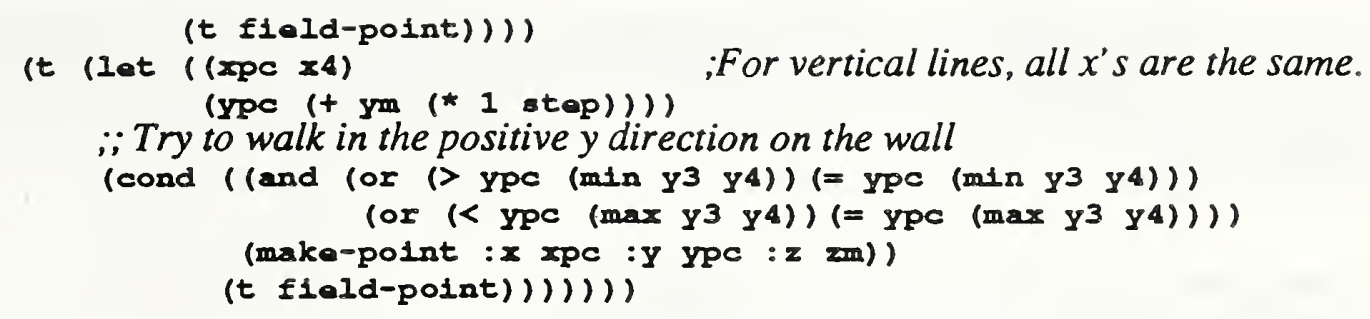

A negative step is just a positive step with a negative step size.

(defun make-new-field-point-negative-step

(exposed-wall field-point step $\beta 2$ )

(make-now-field-point-positive-step

exposed-wall field-polnt ( $*-1$ step) B2))

The following two functions are used above to search either in the positive or negative directions for the maximum.

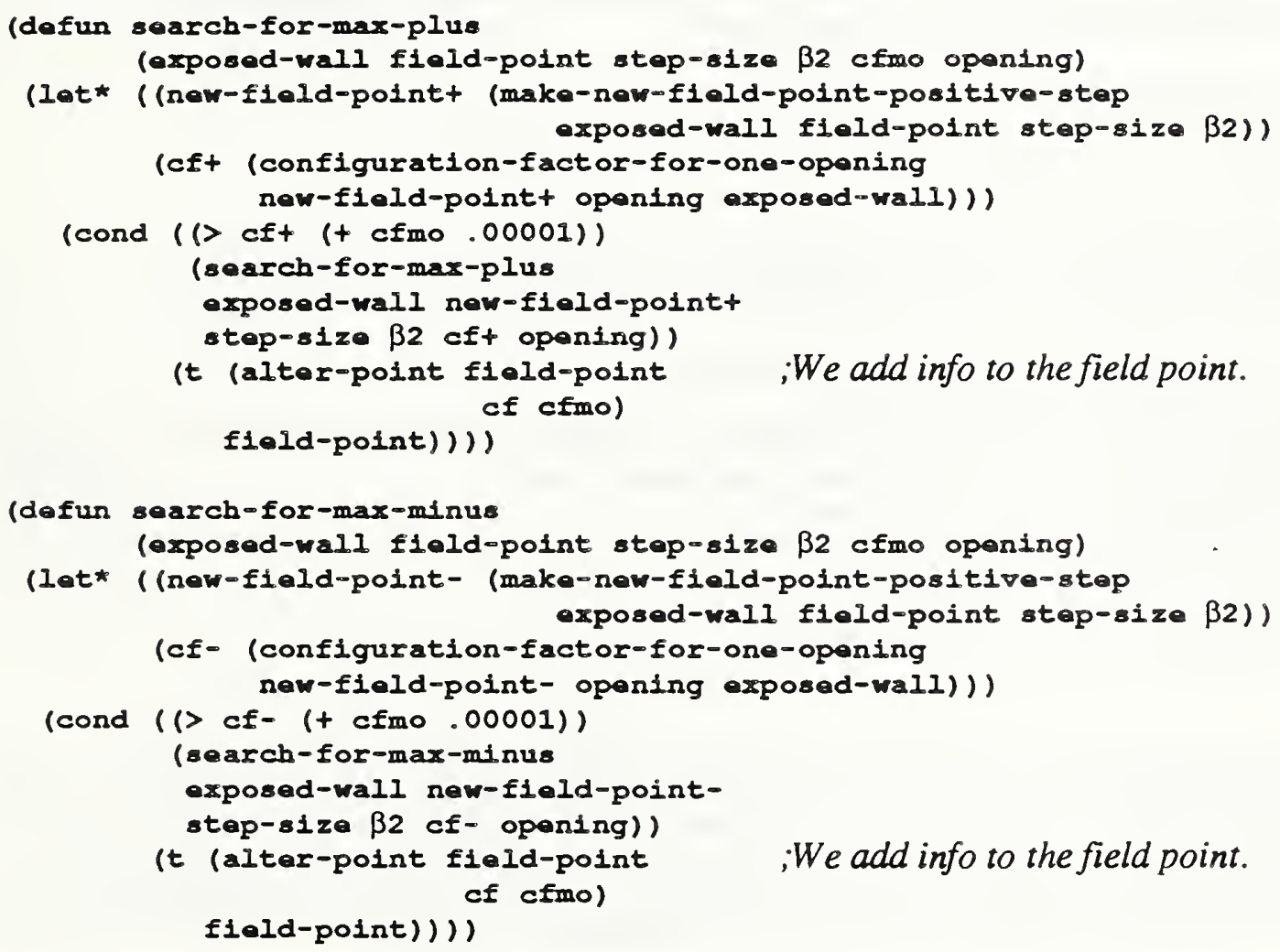

\section{Determine Area to Scan for Maximum}

We now wish to determine the area to scan to determine the maximum configuration factor in some region of an exposed wall. We note that the radiation pattern due to a single opening is smooth and has a single peak. The radiation from more than one opening adds incoherently, i.e., their intensities add. Therefore, a little reflection leads one to conclude that the maximum due to openings will lie inside the area determined by wrapping a string around pegs placed at the locations of the individual opeinings' maximums. However, rather than using the area determined with the pegs and string we will determine a rectangular region that just caputures all the maximums. In some cases, such as for openings, there will be smaller rectangular regions excuded from the region to be scanned. 
We begin by defining a function to collect the location of all the maximums of the individual openings.The following function takes as input the list-of-exposing-openings and the exposed-wall and retums a list of the maximum point for the openings. The slot values for these points includes their location and configuration factor.

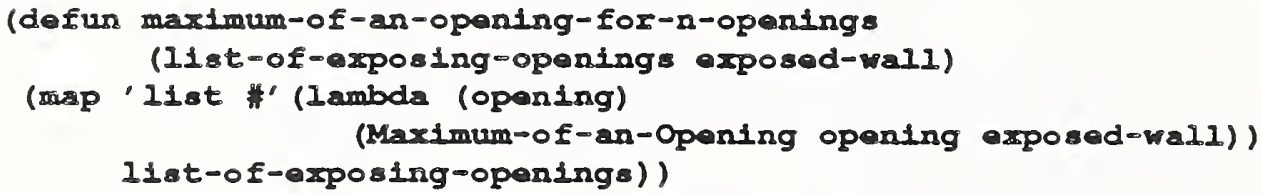

We will have use for the function sub-11st which take as input an element and a 11st-of-11st. It returns a list of all the sub-lists that has element as a member.

(defun sub-11st (alement $11 s t-o f-11 s t$ )

(ramovo alemant list-of-list :tast-not "mamber))

We need two functions. The first determines the separations between the projections into the $x y$ plane of a-point and each point in a list-points.

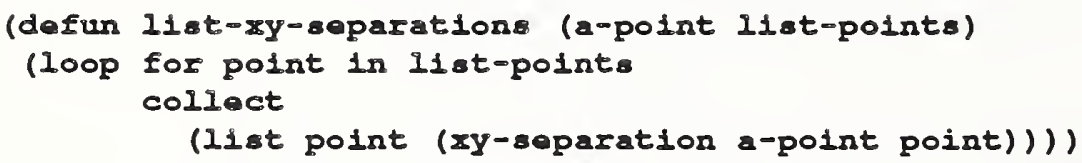

The second function, min-soparation-xy, uses I1st-xy-separations to determine which point in 11st-points which has the smallest xy-separation from a-point.

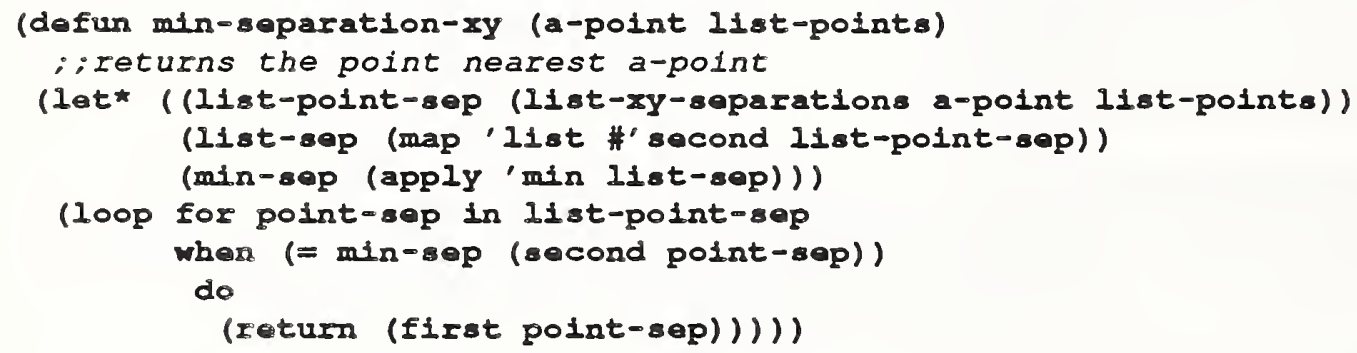

For a region which has no openings in it we proceed as follows. From the list produced by maximum-of-an-opening-for-n-openings, we select out the smallest and the largest values for $z$. Then we use $x y$-separation to determine the points nearest the projection of the lower left and upper right corners of the exposed region. This then defines a rectangular area to scan that will include the maximum of the openings. We used the rectangular area rather than the area determined by wrapping a string around the pegs because it was easier to determine.

The rectangular regions with excluded region or the holey-rectangular region consists of a rectangular region with rectangular holes cut in it. If a maximum falls into an opening, we need to find a replacement maximum for this errant maximum. This replacement maximum will lie on the boundary of the the opening which the original maximum fell into. Thefore, the region we scan is the rectangular region as determined for a nonholey region with the scanning area expanded to include the opening.

To determine if a maximum falls in an opening, we need a function that determines whether a point lies in the interior of a rectangle. The function point-in-rectangle returns the vertical-rectangle if the point lies in the rectangle and nil if not. 


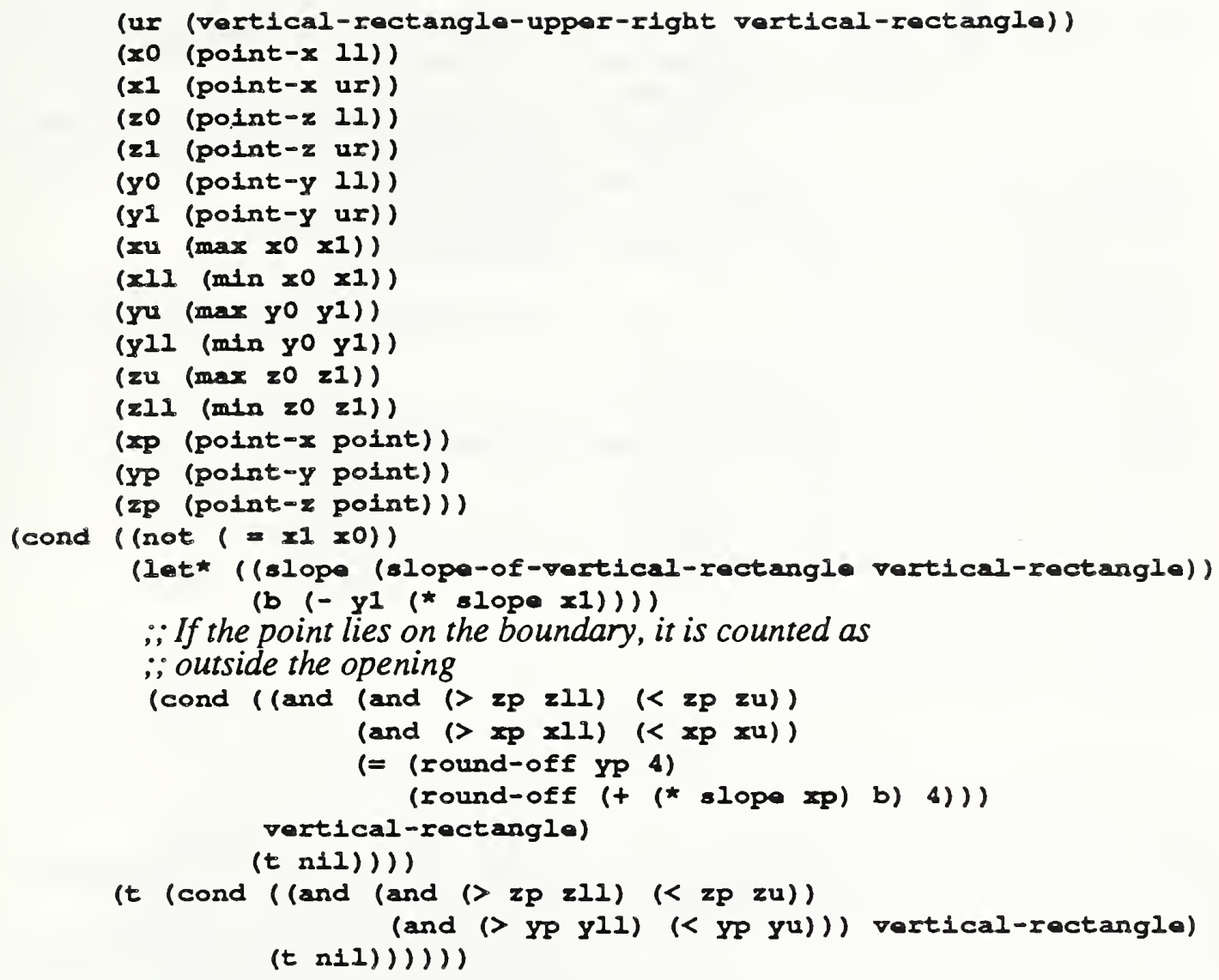

Having the results for one opening, we wish to determine if a point lies in any of a list of openings.

(defun point-in-an-opening (list-of-openings point)

(loop for oponing in list-of-openings do

;A point can only be in only one opening.

(cond ( (point-in-rectangle opening point) (return (point-in-rectangle opening point)))) finally (return nil))

Now suppose we have a list of points and a list of openings and we want a list of openings that have at least one of the points in it.

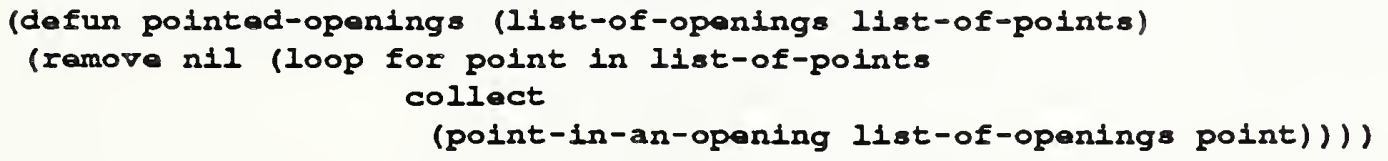

If we have a list of rectangles, we want to generate a list of the lower left and upper right corners.

(defun list-of-comers (11st-of-rectangles)

(append

(map ' list \#' vertical-rectangle-lower-left list-of-rectangles)

(map ' list \#' vortical-rectangle-upper-right list-of-rectangles)))

We are now in a position to determine the area to be scanned on a wall which has openings in it. 
(11st-of-exposing-opentngs exposed-wall list-of-exposed-openlngs)

let* ( (11st-of-max (maxlmum-of-an-opening-for-n-openings

list-of-exposing-openings exposed-wal1))

(11st-of-cornere (polnted-openings 11st-of-axposed-openings

11 st-of-max) )

iIf 11st-of-conners is nil, appond does not add it to the list.

(I1st-of-max-plus (appond 11st-of-max 11st-of-corners))

(11at-z (map 'Iist " "polnt-z I1st-of-max-plus))

(zI (apply' mis Iist-z))

(z2 (apply' $\max 11 \mathrm{~s}-\mathrm{z}-\mathrm{z})$ )

(11 (wall-lower-left axposed-wall))

(ur (wall-upper-right exposed-wall))

(polnt-noar-11 (min-soparation-xy 11 list-of-max-plus))

(polnt-noar-ur (min-aoparation-xy ur liat-of-max-plus))

(n-lower-loft (mako-point : $x$ (point-x point-noar-11)

$: Y($ point-y point-near-II) : $z$ zl))

(n-uppar-right (mako-polnt : $x$ (point-x point-noar-ur)

$: y($ point-y point-noar-ur) :z z2)))

(make-vertical-ractanglo

: Lowar-left n-lowar-left

: uppor-right $n$-upper-right)) )

6. Maximum Configuration Factor for each subregion of the exposed wall.

We wish to determine the maximum radiation field (or configuration factor) for each subregion of the exposed wall. The size of fields we are interested in are those fields that can produce piloted ignition. If the radiation field is small relative to that required for piloted ignition, we are not interested in it. We can define small by looking for the most vulnerable material that might reasonably be exposed. The most vulnerable combustible target is normally assumed to be oven dried wood [5]. If the maximum possible field is below the piloted ignition level for this material then it is assumed that no exposure problem exists.

To take advantage of this assumption, we define a global constant

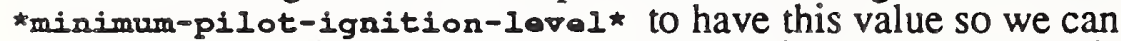
use it as the default value for the piloted ignition level for materials.

(defrar *minimum-pilot-ignition-level* 1.255

"Minimum piloted ignition leval for oven dried wood in $W . / \mathrm{cm}$. sq.")

To determine the maximum configuration factor we can scan the entire subregion of interest and look for the maximum. However, calculating the configuration factor for all these points of the scan takes a lot of computer computation time. Therefore, it is desirable to hold the number of points we scan to some reasonable minimum. This is achieved by scanning over the area in steps as large as possible, but small enough so we don't make a serious error in determining the maximum.

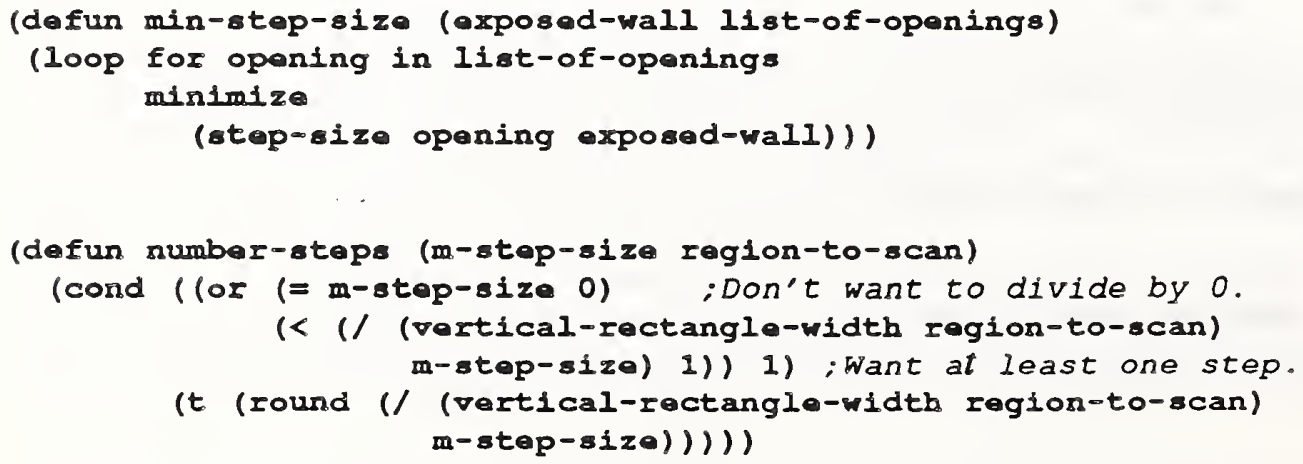

The maximum configuration factor due to a number of openings is always less than the sum 
of the maximums of the individual openings. We will have use for this sum so the following function computes it.

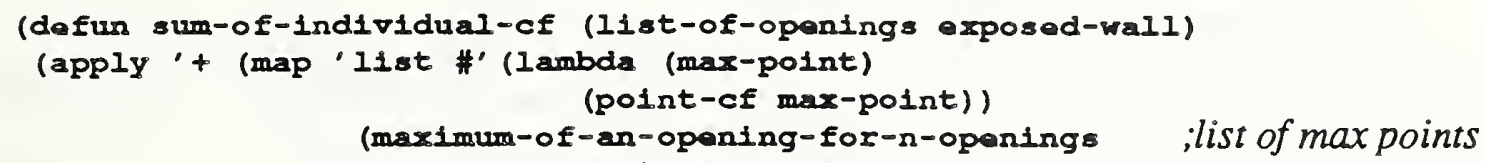

We will scan the wall by using the vertical scan to handle the horizontal scan. If a point falls in an opening, it will be excluded.

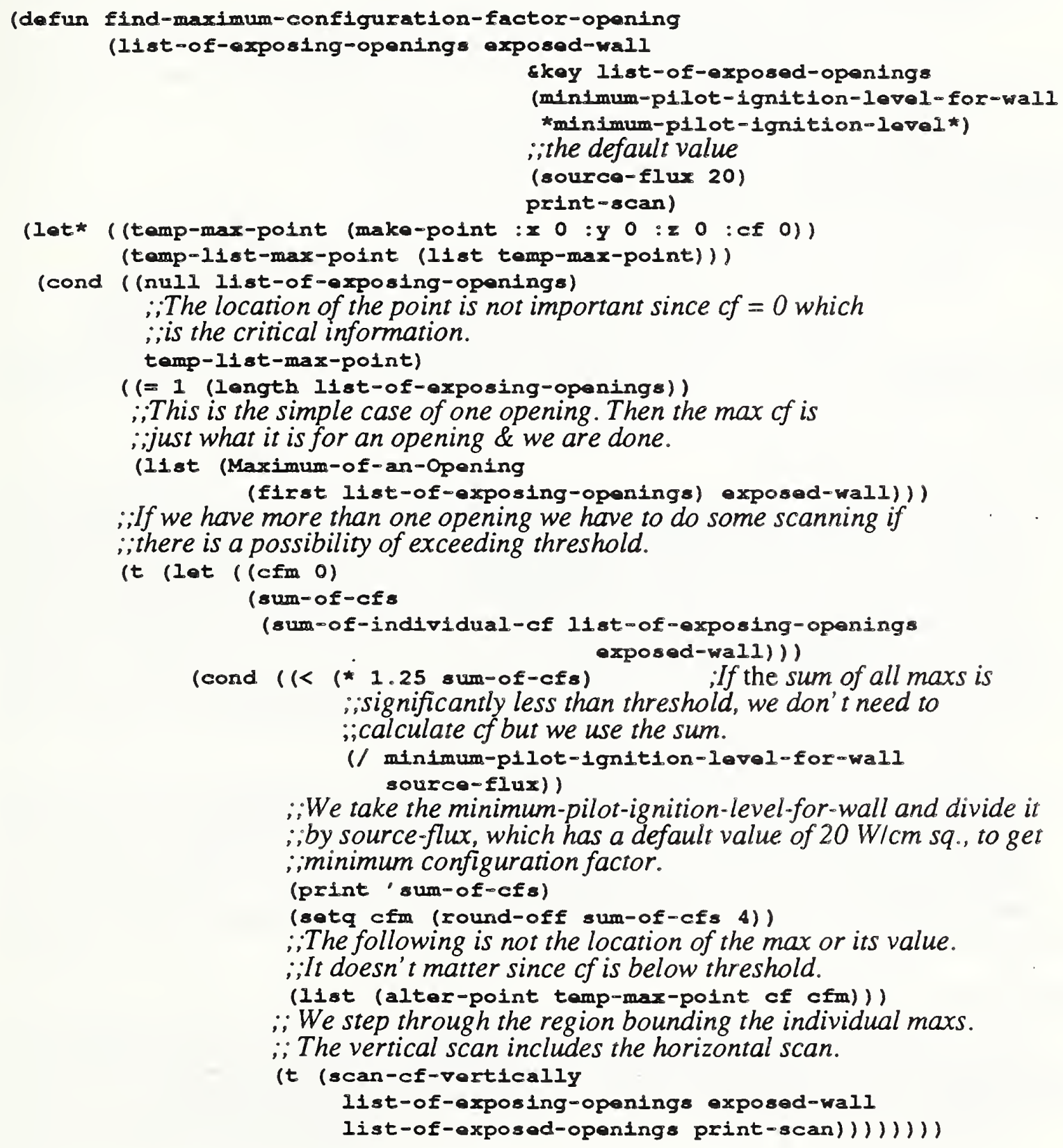

The following function scans the horizontal scanning function vertically.

(defun scan-cf-vertically

(list-of-exposing-openings exposed-wall

list-of-exposed-openings print-scan) 


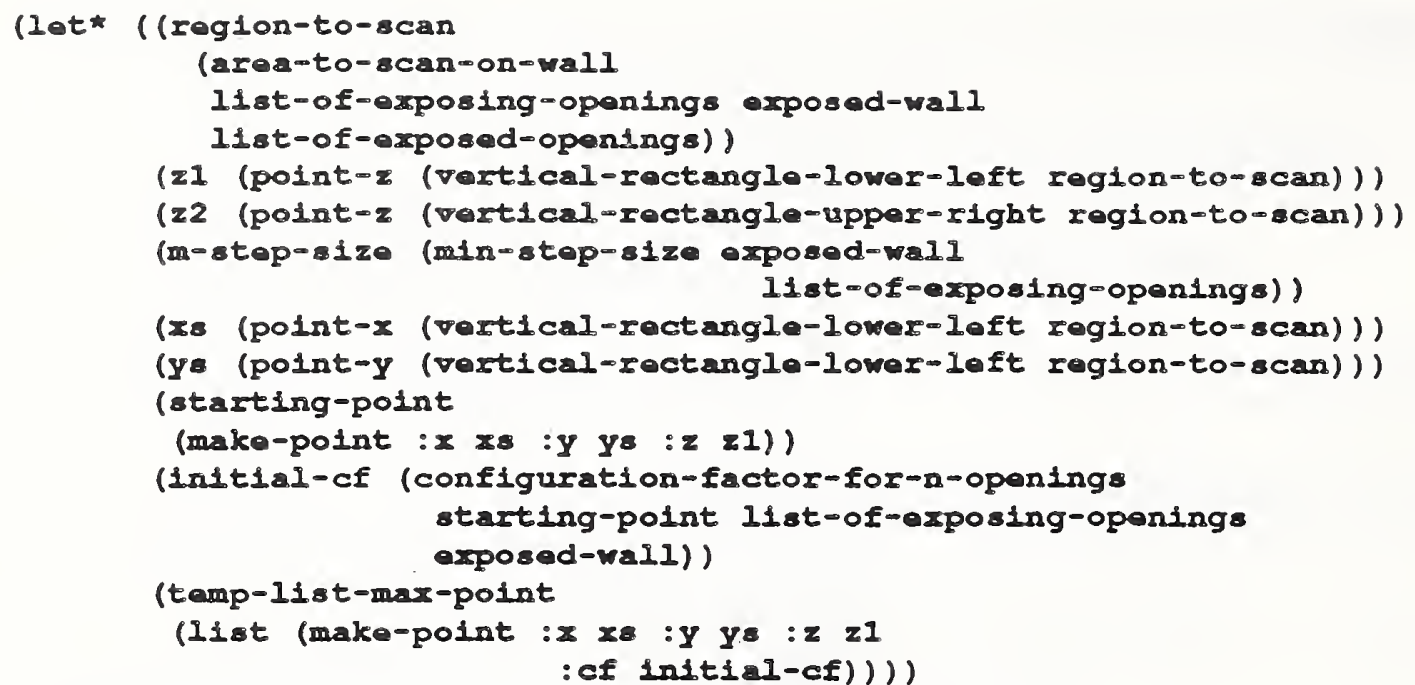

\#For diagnostic purposes it is sometimes of interest to print out

$\because$ ithe area to be scanned.

(cond (print-scan

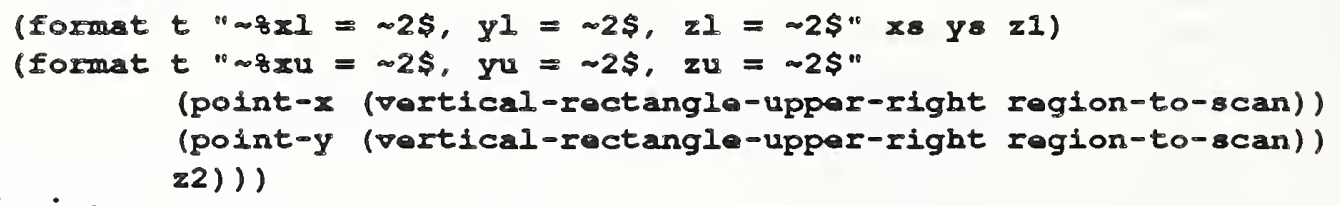

;Back to business.

(loop for $z$ from $z 1$ to $z 2$ by m-stop-sizo

do

(setg temp-11st-max-point

(scan-ef-horizontally

z list-of-oxposing-oponings exposed-wall

list-of-exposed-openinga temp-list-max-point

m-stop-size rogion-to-scan print-scan))

finaliy (roturn tomp-1ist-max-point))))

The following is the horizontal scanning function.

(defun san-cf-borizontally

;We fix $z$ and scan over $x y$.

(z list-of-exposing-openings oxposed-wall

11st-of-exposed-openings temp-list-max-point

m-step-size ragion-to-scan print-scan)

(let* ( $x$ (point-x (vertical-rectangle-lower-left region-to-scan)))

(y (point-y (vertical-rectanglo-lower-left region-to-scan)) )

(field-point (make-point : $x$ x :y $y: z \quad z)$ )

( $\beta 2$ (alope-of-wall oxposed-wall))

(number-steps (number-steps m-step-size region-to-scan)))

(loop for $k$ from 0 to number-steps

do

(alter-point field-point

$$
\begin{aligned}
& x(+x(* k m-s t e p-s i z o(\cos \beta 2))) \\
& Y(+y(* k m-s t e p-s i z o(\sin \beta 2))))
\end{aligned}
$$

"ilf field-point is in an opening, we skip it.

(cond ((point-in-an-opaning list-of-oxposad-opanings field-point)) $\because$ Otherwise we process it.

(t) $(10 \mathrm{t}$

( naw-cf

(round-off (configuration-factor-for-n-openings field-point list-of-exposing-openinga exposed-wall) 4))

(current-max-cf (point-cf (first 
;If we make print-scan true, we get a printout of the scan.

(cond (print-scan

(format

$t$

$" \sim 8 x=\sim 2,0,6 \$, y=-2,0,6 \$, z=\sim 2,0,6 \$$, new-cf $=\sim 4,0,6 \$, \mathrm{~m}-$

stop-size $=-2,0,6 \$ "$

(point-x field-point) (point-y field-point) (point-z fie

Id-point)

(cond ( $(>$ now-cf current-max-cf), ;replace entire list

(sotq tomp-list-max-point

(list (make-point :x (point-x fiold-point)

:y (point-y field-point)

$: z$ (point-z field-point)

:(c) now-cf)) )l

$((=$ now-cf curront-max-cf) ;add to list

(sotq tomp-list-max-point

(cons (make-point :x (point-x field-point)

$: y$ (point-y field-point)

$: 2$ (point-z field-point)

:cf now-cf)

tamp-1ist-max-point)))l))

finally (return temp-list-max-point))))

To convert to field values from configuration factors we multiply configuration factors by the source's flux. This is such a simple thing we do not need a function to compute it.

\section{Conclusions and Possible Future Work}

This report documents a program module in the expert system EXPOSURE. It is used for calculating the maximum electromagnetic radiation incident upon a building's wall due to the burning of a neighboring building. It is assumed that the burning building has an arbitrary number of rectangular openings emitting radiation. The exposed wall can be considered as being composed of a number of rectangular regions. These regions may be openings or regions of different material. The results of calculating the configuration factor using the function configuration-factor-for-one-opening with analytical expressions for three special cases showed agreement to 4 or 5 decimal places (see Appendix A). This is just little more accuracy that the expert system requires so that is desirable.

This module also allows the consideration of nonparallel exposing and exposed walls and the explicit use of the appropriate wall failure mechanism. In the case of combustible walls, this means using the piloted ignition threshold for the combustible material rather than a very conservative value for all combustible materials such as is done by NFPA 80A. Also, the effect of the location of opening is taken into consideration. Again this is beyond current practice. These features improve the accuracy of the analysis of the safety of buildings as it relates to the spread of fire between buildings and facilitates innovative design and use of materials.

Future work could include work on the selection of the step size used in scanning. In many real world problems an exposing building with many windows exposes a long wall. The present program could probably be made faster by a more intelligent selection of the initial step size and increasing the step size in regions of small configuration factors. Also, much more effort needs to be spend on testing the program before it could be considered being used in a deployed expert system. 


\section{References}

1. Routley, Gordon "Fire Department Operations" Cote, Arthur E. and Jim L. Linville, Fire Protection Handbook, 16 Ed. National Fire Protection Association, Mass. 1986 pp 15-23 to 15-36.

2. NFPA 80A: Recommended Practice for Protection of Buildings from Exterior Fire Exposures. [1980]

3. Protection Against Fire Exposure, Loss Prevention Data 1-20, March 1979, Factory Mutual System

4. McGuire, J. H. "Fire and the Spatial Separation of Buildings." Fire Technology. 1(4): 278-2871965.

5. Law, Margaret, "Heat Radiation from Fires and Building Separation" Joint Fire Research Organization Technical Paper no. 5, 1963

6. Willians-Leir, G. "Approximations for Spatial Separation" Fire Technology, vol 2 no. 2 136-1451966

7. Drysdale, Dougal. An Introduction to Fire Dynamics. New York: John Wiley and Sons 1985.424 p.

8. Siegel, Robert and John R. Howell, Thermal Radiation Heat Transfer. 2nd Ed. McGraw-Hill Book Co. 1981

9. Tein, C. L., K. Y. Lee, and A. J. Stretton "Radiation Heat Transfer" SFPE Handbook of Fire Protection Engineering, 1st Ed. pp 1-93 to 1-106 National Fire Protection Association, Mass 1988 
Appendix A: Configuration Factor Calculation Compared to Results of Using an Analytic Expression

In this appendix we will compare the program's results for the configuration factor of a single opening with results obtained by the use of analytical expressions for three special cases.

Case 1.

The first special case is when the infinitesimal element is parallel to the opening and located on a line passing through a corner of the opening and the line is perpendicular to the plane of the opening.

The analytical expression which we obtained from reference 8 page $823 \# 4$, is expressed in Lisp code in the function cf-parallel-corner.

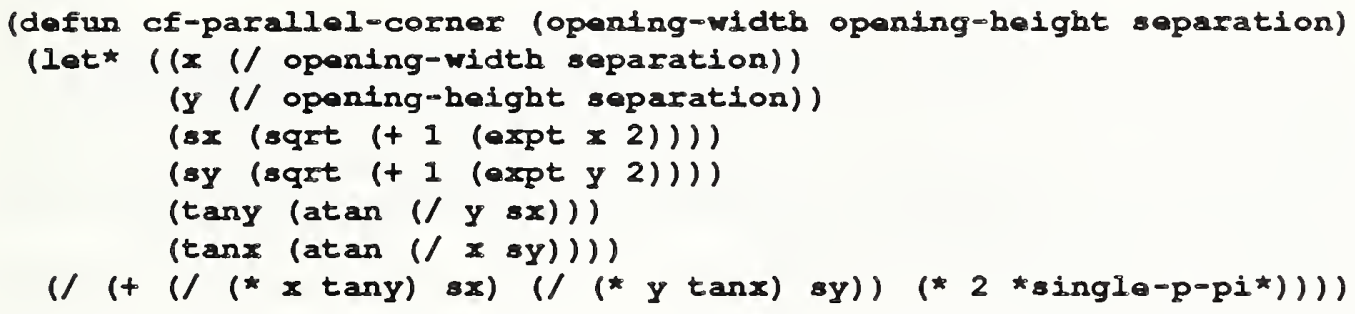

We write the following function to compare the program's result with that obtained by using cf-parallel-corner.

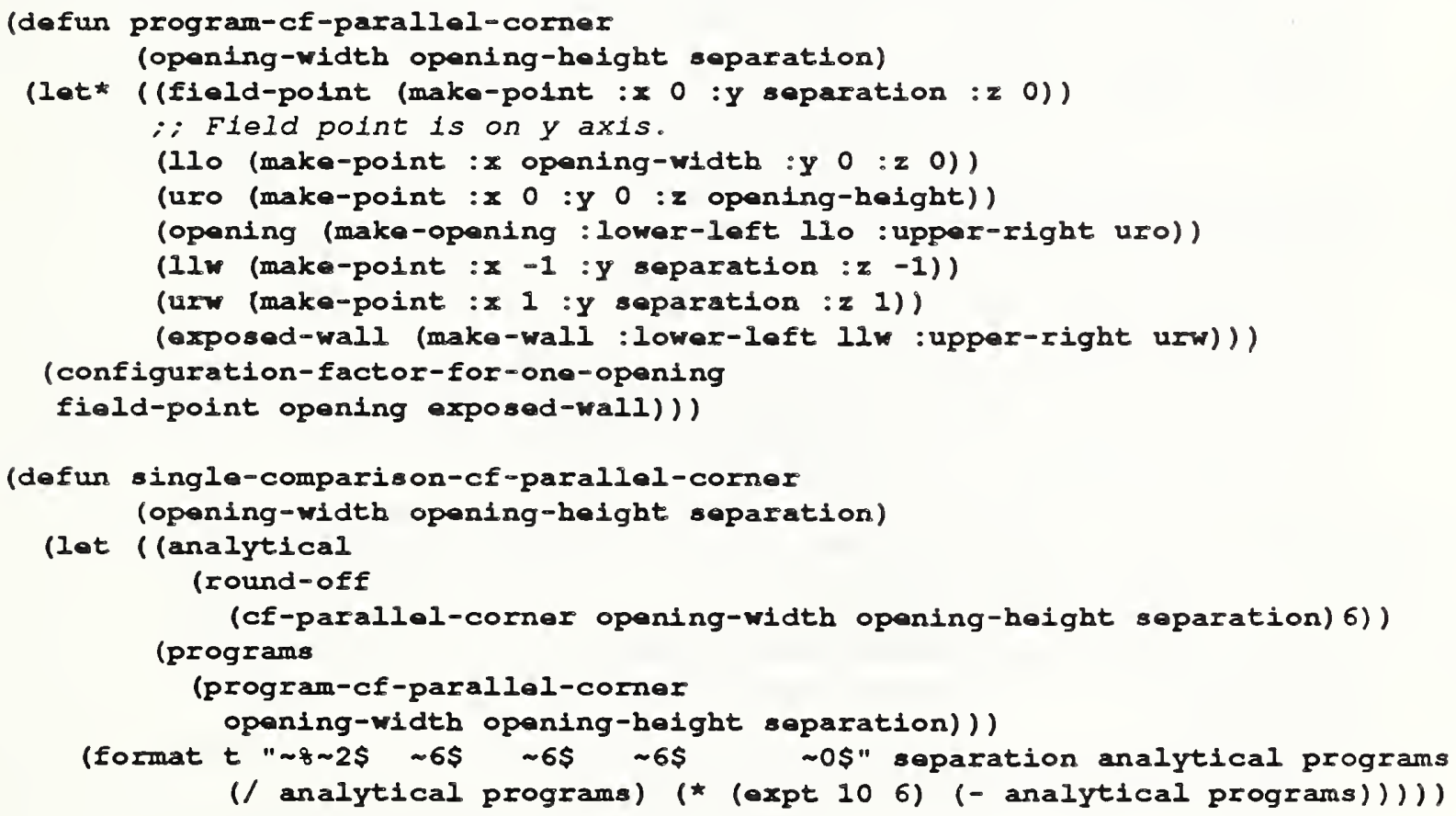

If we run the function

(IIst-comparison-cf-parallel-corner' (.25 .5 1234567 )) 
with the indicated arguments, we obtain:

$\begin{array}{lclcl}Y & \text { analytical } & \text { program } & \text { analyt./prog. } & \text { difference } \times 10 \wedge 6 \\ 0.25 & 0.237856 & 0.237857 & 0.999995 & -1 . \\ 0.50 & 0.207757 & 0.207767 & 0.999954 & -10 . \\ 1.00 & 0.138532 & 0.138572 & 0.999710 & -40 . \\ 2.00 & 0.059864 & 0.059909 & 0.999255 & -45 . \\ 3.00 & 0.030829 & 0.030864 & 0.998858 & -35 . \\ 4.00 & 0.018369 & 0.018390 & 0.998866 & -21 . \\ 5.00 & 0.012089 & 0.012105 & 0.998641 & -16 . \\ 6.00 & 0.008527 & 0.008535 & 0.999078 & -8 . \\ 7.00 & 0.006324 & 0.006329 & 0.999242 & -5 .\end{array}$

\section{Case 2.}

Configuration factor perpendicular over one corner

The second special case is when the infinitesimal element is perpendicular to the opening and located on a line passing through a corner of the opening and the line is perpendicular to the plane of the opening.

The analytical expression which we obtained from reference 8 page $823 \# 6$, is expressed in Lisp code in the function of -perpendicular-corner.

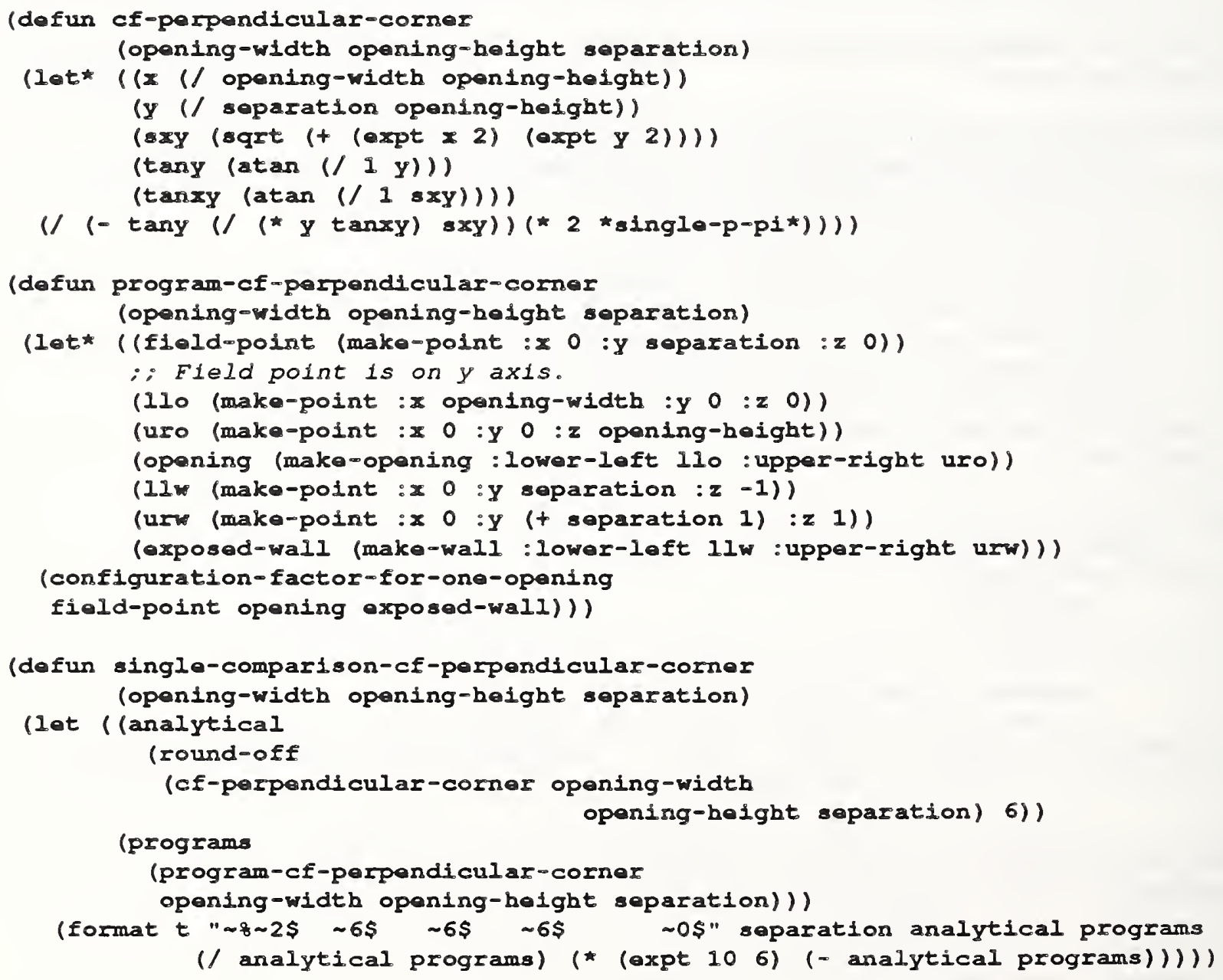


(format $t " \sim 8 Y$ analytical program analyt./prog. difference $x 10 \wedge 6 "$ )

lloop for $y$ in list-of-separations do

(single-comparison-cf-perpendicular-corner 1 I y))

If we run the function

(Ilst-comparison-cf-perpendicular-corner' $\left(\begin{array}{llll}.5 & 1 & 2\end{array}\right)$

with the indicated arguments, we obtain:

$\begin{array}{lclcl}Y & \text { analyt1cal } & \text { program } & \text { analyt./prog. } & \text { dffference } \times 10^{\wedge} 6 \\ 0.50 & 0.124269 & 0.124326 & 0.999541 & -57 . \\ 1.00 & 0.055734 & 0.055789 & 0.999010 & -55 . \\ 2.00 & 0.013928 & 0.013952 & 0.998309 & -24 . \\ 3.00 & 0.004964 & 0.004976 & 0.997573 & -12 .\end{array}$

Case 3.

Configuration factor angle theta over one comer

The third special case is when the infinitesimal element is in a plane that has an angle of intersection with the plane of the opening of theta. The separation is the distance from the corner of the opening where the two planes intersect to the infinitesimal element.

Reference "Fire-Safe Structural Steel A Design Guide" American Iron and Steel Institute, (1979) page 24

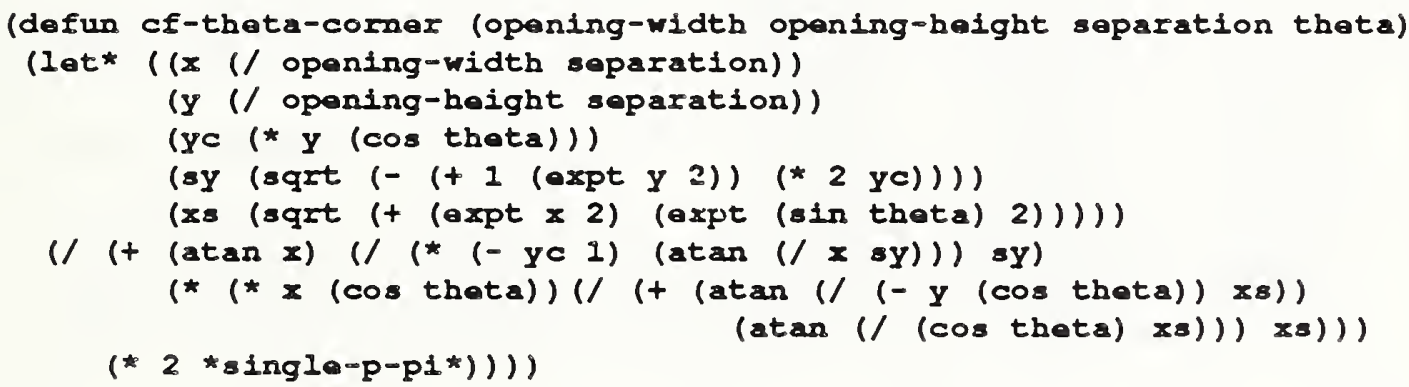

(dofun program-cf-thota-cornex

(opening-width opening-height separation thota)

(let* ((field-point (make-point :x (* separation (cos theta))

$: y$ (* separation ( $s i \Omega$ theta))

$: 20)$ )

(1I0 (make-point :x opening-width:y $0: z 0)$ )

(uro (make-point :x $0: y 0: z$ opening-height))

(opening (make-opening : lower-left llo :upper-right uro))

(IIw (make-point :x (* soparation (cos theta))

:y (* soparation (sin thota))

$(2-1))$

(urw (make-point :x (* (t separation 1) (cos theta))

$: y$ (* (t soparation 1$)$ (sin thota))

$: 21)$

(exposed-wall (make-wall : lower-left IIw :upper-right urw)))

(configuration-factor-for-one-opening

field-point opening exposed-wall)))

(defun single-comparison-cf-theta-corner

(opening-width opening-height separation theta)

let ( (analytical

(round-off

(cf-theta-corner opening-width 


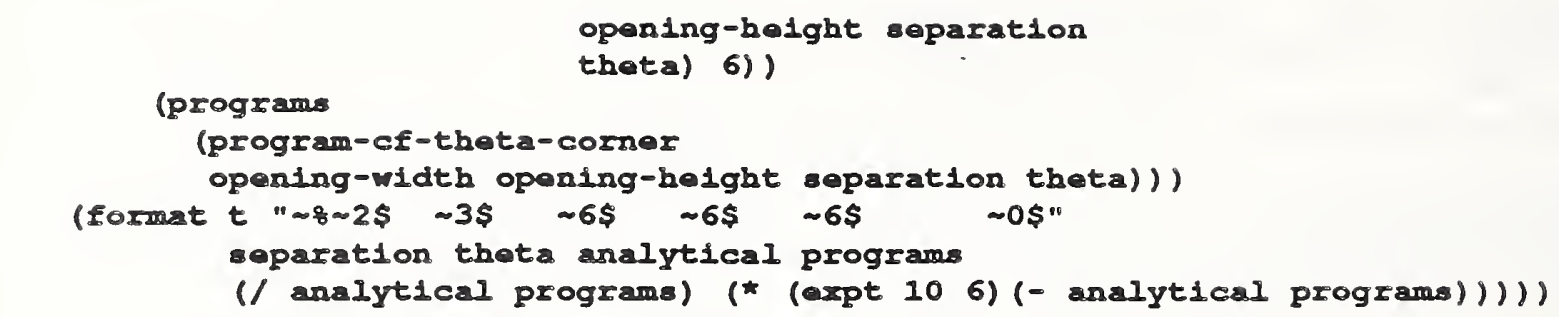

(defun 11st-comparison-cf-thota-cornor

(Ilat-of-separations 11st-of-tbotas)

(format $t$ " $\sim 8$ thota analytical program analyt./prog. differonce xion6")

(loop for $x y$ in list-of-soparations

do

(loop for thota in list-of-thotas

do

(singlo-comparison-cf-tbeta-corner $11 \mathrm{xy}$ thota)))

If we run the function (11st-comparison-cf-thota-comer ' $\left(\begin{array}{lll}1 & 2 & 3\end{array}\right)$ (11st (/ * single-p-pi* 2) (* 3 (/ *aingle-p-pi* 4)) (* 3.5 (/ *aingle-p-p1* 4)) (* $3.9(/ \star s i n g l e-p-p 1 * 4)))$ ) with the indicated arguments, we obtain:

$\begin{array}{lcclcl}Y & \text { theta } & \text { analytical } & \text { program } & \text { analyt./prog. } & \text { difference x10^6 } \\ 1.00 & 1.571 & 0.055734 & 0.055789 & 0.999010 & -55 . \\ 1.00 & 2.356 & 0.013020 & 0.013042 & 0.998303 & -22 . \\ 1.00 & 2.749 & 0.003201 & 0.003207 & 0.997986 & -6 . \\ 1.00 & 3.063 & 0.000127 & 0.000128 & 0.995048 & -1 . \\ 2.00 & 1.571 & 0.013928 & 0.013952 & 0.998309 & -24 . \\ 2.00 & 2.356 & 0.003797 & 0.003810 & 0.996505 & -13 . \\ 2.00 & 2.749 & 0.000961 & 0.000965 & 0.996236 & -4 . \\ 2.00 & 3.063 & 0.000039 & 0.000039 & 1.007274 & 0 . \\ 3.00 & 1.571 & 0.004964 & 0.004976 & 0.997573 & -12 . \\ 3.00 & 2.356 & 0.001542 & 0.001552 & 0.993681 & -10 . \\ 3.00 & 2.749 & 0.000401 & 0.000403 & 0.993925 & -2 . \\ 3.00 & 3.063 & 0.000016 & 0.000016 & 0.980120 & -0 .\end{array}$


Appendix B: Results for Some Simple Buildings

We present here the results of applying the results of this file to three cases that will test the performance of this program. It is not a comprehensive test, but one that should exercise the program's main features.

We define a function that will take as its first argument a list of the list of lower left and upper right corners of the openings. The next six arguments are the coordinates of the lower left and upper right comers of the exposed area.

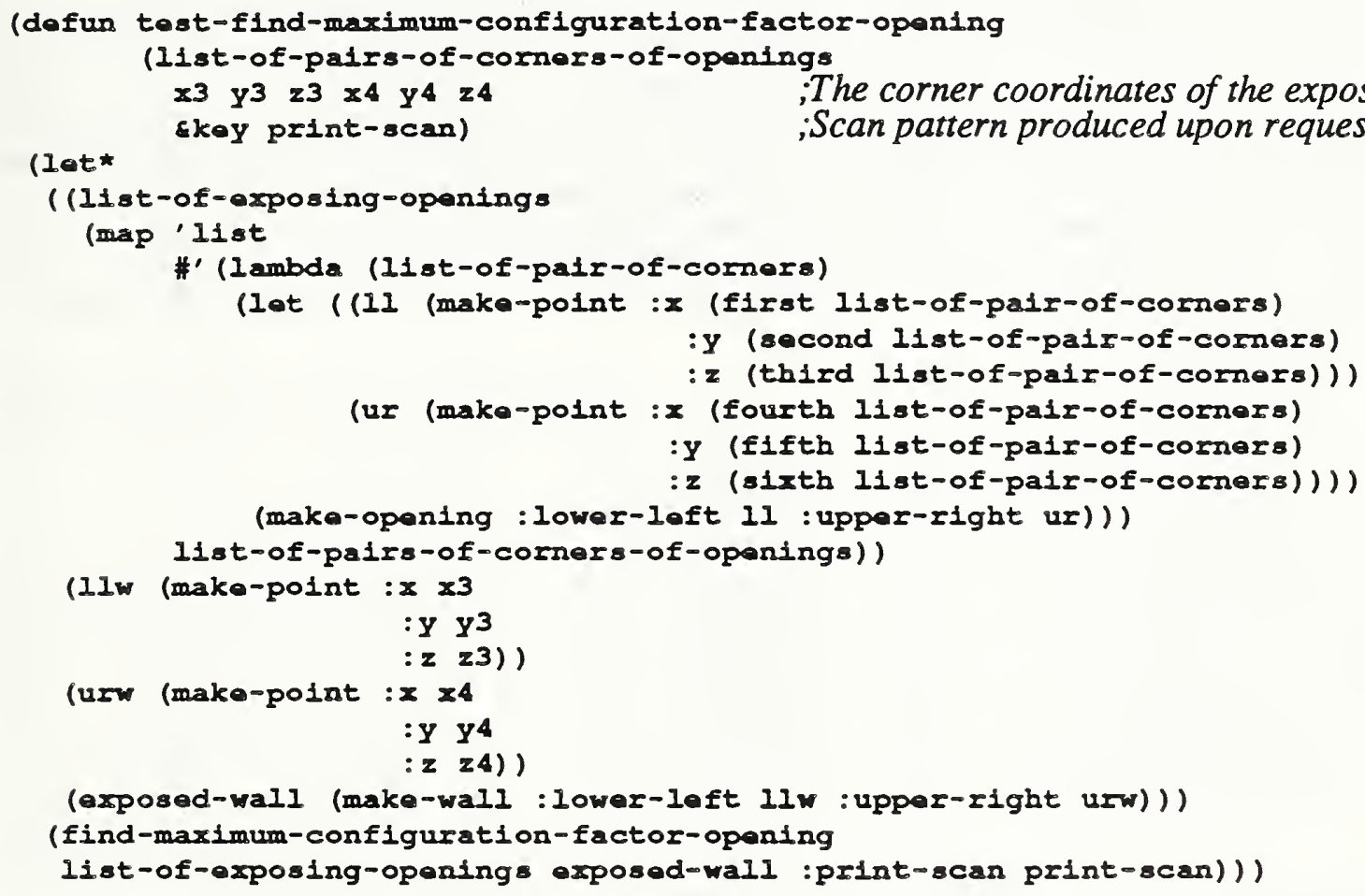

We will test three cases. In all cases we have two exposing windows either in the $\mathrm{xz}$ or $\mathrm{yz}$ plane. While the windows are the same size, they are set in at different heights. For the first two cases the exposed wall is parallel to the exposing wall. In the last case, it is at a small angle to the exposing wall.

Our first test examines the time need to compute the results.

(defun test-cases ()

(values (time (test-find-maximum-configuration-factor-opening

(tarpri)

$$
\cdot\left(\left(\begin{array}{lllllllllllll}
0 & 1 & 1 & 0 & 7 & 7
\end{array}\right)\left(\begin{array}{llllllll}
0 & 9 & 7 & 0 & 15 & 13
\end{array}\right)\right.
$$

$\therefore$ Interchanging $x$ and $y$, we get

(time (test-find-maximum-configuration-factor-opening

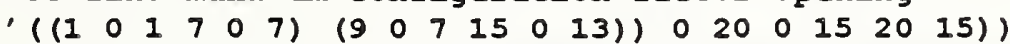

(tarpri)

$\therefore$ Setting the exposed wall at a small angle to the exposing wall.

(tima (test-flnd-maximum-configuration-factor-opening

$\left(\left(\begin{array}{lllllllllllll}1 & 0 & 1 & 7 & 0 & 7\end{array}\right)\left(\begin{array}{lllllllllll}9 & 0 & 7 & 15 & 0 & 13\end{array}\right)\right.$

The results of running test-cases follows:

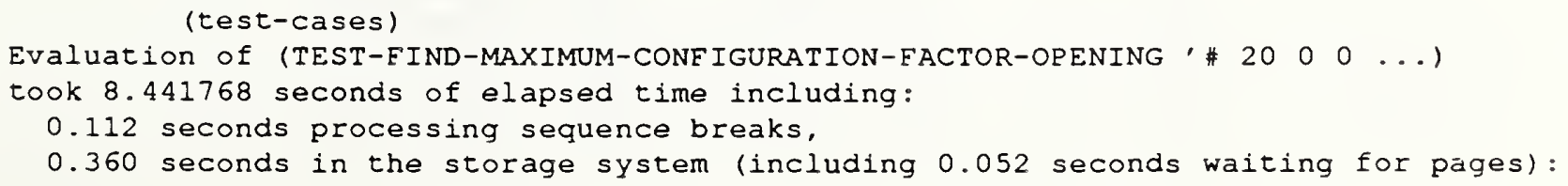


0.179 seconds processing 313 page faults including 4 fetches,

0.171 seconds in creating and destroying pages, and

0.010 seconds in miscellaneous storage system tasks.

The garbage collector has flipped; so no consing was measured.

Evaluation of (TEST-FIND-MAXIMUM-CONEIGURATION-FACTOR-OPENING ' \# $0200 \ldots$ )

took 6.812716 seconds of elapsed time including:

0.137 seconds processing sequence breaks,

0.531 seconds in the storage system (including 0.135 seconds waiting for pages):

0.312 seconds processing 330 page faults including 21 fetches,

0.159 seconds in creating and destroying pages, and

0.060 seconds in miscellaneous storage system tasks.

235 Ilst, 40,365 structure, 12,325 stack words consed in WORKING-STORAGE-AREA.

Evaluation of (TEST-FIND-MAXIMUM-CONEIGURATION-FACTOR-OPENING $\quad$ \# $0180 \ldots \ldots$ )

took 8.779506 seconds of elapsed time including:

0.173 seconds processing sequence breaks,

0.587 seconds in the storage system (including 0.082 seconds waiting for pages):

0.220 seconds processing 391 page faults including 6 fetches,

0.231 seconds in creating and destroying pages, and

0.135 seconds in miscellaneous storage system tasks.

221 list, 44,207 structure, 18,121 stack words consed in WORKING-STORAGE-AREA.

(\#SIPOINT :X 20.0

$: Y \quad 6.828427$

: 2.828427

:CF $247 / 5000)$ )

NII

(\#SIPOINT : X 6.828427

$:$ Y 20.0

: 6.828427

:CF 247/5000))

NII

(\#S (POINT :X 2.5196323

:Y 18.671902

:Z 6.607681

:CF 513/10000))

The second shows the area to be scanned and the actual scanned points.

(defun test-cases-2 ()

(values (test-find-maximum-configuration-factor-opening

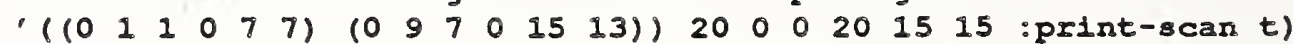

(torpri)

$\therefore$ Interchanging $x$ and $y$, we get

(test-find-maximum-configuration-factor-opening

$\left(\begin{array}{llllllllll}1 & 0 & 1 & 7 & 0 & 7\end{array}\right)\left(\begin{array}{llllllllll}9 & 0 & 7 & 15 & 0 & 13\end{array}\right) \quad 0 \quad 200152015$ :print-scan $\left.t\right)$

(torpi1)

$\therefore$ Setting the exposed wall at a small angle to the exposing wall.

(test-find-maximum-configuration-factor-opening

$\left(\begin{array}{llllllll}1 & 0 & 1 & 7 & 0 & 7\end{array}\right)\left(\begin{array}{llllll}9 & 0 & 7 & 15 & 0 & 13\end{array}\right)$

0180152215 :print-scan t))

The results of running test-cases-2 follows:

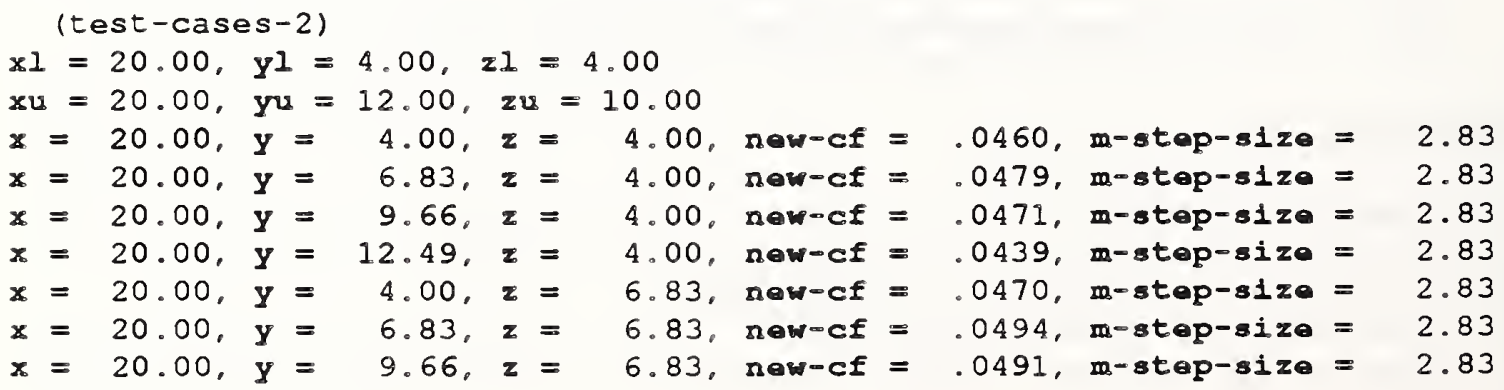




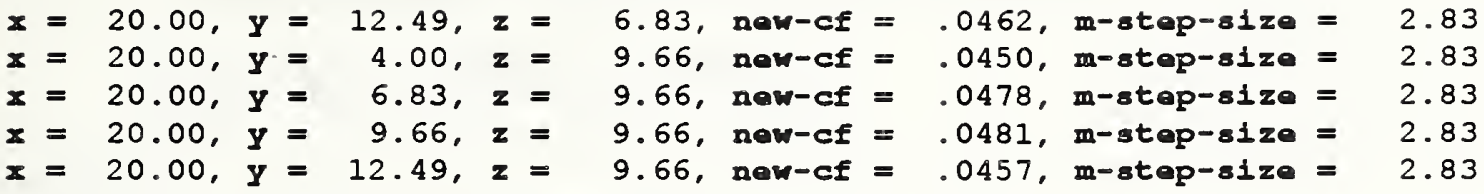

$z I=4.00, y^{I}=20.00, \quad z I=4.00$

$\mathbf{x u}=12.00, y u=20.00$, zu $=10.00$

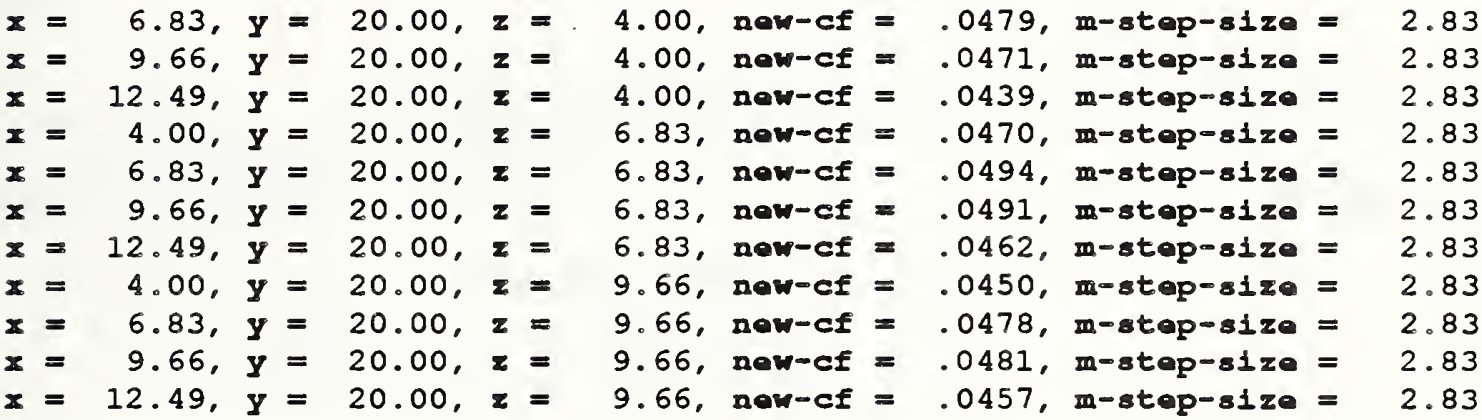

$x I=0.00, \quad y^{1}=18.00, \quad z 1=4.00$

$x u=6.72, y u=19.79, z u=10.00$

\begin{tabular}{|c|c|c|c|c|c|c|c|c|c|c|c|c|c|}
\hline$=$ & .00 & $\boldsymbol{Y}$ & $=$ & 18.00 & $\mathbf{z}$ & $=$ & 4.00 & now-cf & $=$ & .0485 , & m-step-sizo & $=$ & 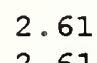 \\
\hline$=$ & 2.52 & $y$ & $=$ & 18.67, & $\mathbf{z}$ & - & 4.00, & new-cf & 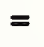 & .0503 & $m-8 t e p-81 z 0$ & $=$ & - \\
\hline$=$ & 5.04, & $y$ & $=$ & 19.34. & $\mathbf{z}$ & $=$ & 4.00, & new-cf & $=$ & .0495. & $m-s t e p-81 z 0$ & $=$ & 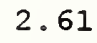 \\
\hline 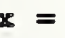 & 7.56 & $\boldsymbol{Y}$ & $=$ & 20.02 , & $\mathbf{z}$ & 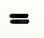 & 4.00 & new-cf & $=$ & .0463 & m-step-size & $=$ & 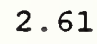 \\
\hline 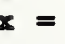 & .00 & $Y$ & $=$ & 18.00, & $\mathbf{z}$ & $=$ & 6.61 , & now-cf & 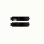 & 90, & $m-s t a p-s i z e$ & 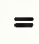 & \\
\hline$=$ & 2.52 & $\boldsymbol{Y}$ & $=$ & 18.67, & $\mathbf{z}$ & $=$ & 6.61 , & now-cf & $=$ & .0513. & $m-s t e p-s 1 z 0$ & $=$ & 2. \\
\hline$=$ & 5.04, & $y$ & $=$ & 19.34, & $z$ & $=$ & 6.61 . & new-cf & $=$ & .0509 & $m-s t e p-s i z e$ & - & 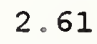 \\
\hline$=$ & 7.56, & $\boldsymbol{Y}$ & $=$ & 20.02 , & $\mathbf{z}$ & $=$ & 6.61 , & now-cf & $=$ & .04 & $m-s t e p-s i z 0$ & $=$ & ?. \\
\hline 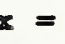 & .00 , & $\boldsymbol{y}$ & $=$ & 18.00, & $\mathbf{z}$ & $=$ & 9.22 & nen-cf & 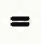 & .0 & $m-s t o p-s i z 0$ & $=$ & 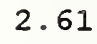 \\
\hline- & 2.52 & $Y$ & $=$ & 18.67, & $\mathbf{z}$ & $=$ & 9.22 & now-cf & $=$ & .04 & $m-s t e p-81 z e$ & $=$ & \\
\hline 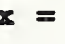 & 5.04, & $\boldsymbol{Y}$ & $=$ & 19.34, & $\mathbf{z}$ & $=$ & 9.22 & new-cf & $=$ & .0495 & m-step-size & $=$ & . \\
\hline- & 7.56 & $\mathbf{Y}$ & $=$ & 20.02 & $\mathbf{z}$ & $=$ & 9.22 & now-cf & $=$ & .0473 & $m-s t e p-s i z e$ & $=$ & 2. \\
\hline
\end{tabular}

(\#S (POINT:X 20.0

: $Y$ 6.828427

: Z 6.828427

:CE 247/5000))

NIL

(\#S (POINT :X 6.828427

$: Y 20.0$

:2 6.828427

:CF 247/5000))

NIL

(\#SIPOINT :X 2.5196323

:Y 18.671902

:2 6.607681

:CF 513/10000)) 


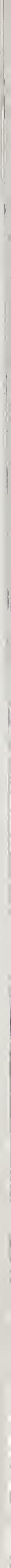




\section{BIBLIOGRAPHIC DATA SHEET}

3. PUBLCATION DATE

November 1990

4. TITLE AND SUBTITLE

A Program for Calculating the Maximum Radiation on a Wall

5. AUTHOR(S)

Richard L. Smith

6. PERFORMING OAGANIZATION (IF JOINT OR OTHER THAN NIST, SEE INSTRUCTIONS)

U.S. DEPARTMENT OF COMMERCE

NATIONAL INSTITUTE OF STANDARDS AND TECHNOLOQY

GNTHERSBURG, MD 20890

9. SPONSORINO ORGANIZATION MAME AND COMPLETE AODRESS (STREET, CITY, STATE, ZIP)

U.S. Air Force Engineering and Services Center

Airbase Fire Protection and Crash Rescue System Branch

Tyndall AFB, FL 32403-6001

10. SUPPLEMENTARY NOTES

11. ABSTRACT (A 200-WORD OR LESS FACTUAL SUMMARY OF MOST SIONIFICANT INFORMATION. IF DOCUMENT INCLUDES A SIGNIFICANT BIBLOOGRAPHY OF UTERATURE SURVEV, MEMTION IT HERE.)

This report describes a program module of the expert system EXPOSURE. This module, written in Common Lisp, is for calculating the maximum electromagnetic radiation incident upon. a building's wall due to theburning of a neighboring building. It is assumed that the burning building has an arbitrary number of rectangular openings emitting radiation. The exposed wall can be considered as being composed of a number of rectangular regions. These regions may be openings on regions of different materials. This program can determine the maximum radiation for each of these regions and for the wall less these regions.

12. KEY WORDS (6 TO 12 ENTRIES; ALPHABETICAL ORDER; CAPITALZE ONLY PROPER NAMES; AND SEPARATE KEY WORDS BY SEMICOLONS) exposure; radiation; radiative h eat transfer; computer programs 


